\title{
Compressed Resolvents and Reduction of Spectral Problems on Star Graphs
}

\author{
B. Malcolm Brown ${ }^{1}$ - Heinz Langer ${ }^{2}$ - Christiane Tretter ${ }^{3}$
}

Received: 26 January 2018 / Accepted: 23 April 2018 / Published online: 22 May 2018

(C) The Author(s) 2018

\begin{abstract}
In this paper a two-step reduction method for spectral problems on a star graph with $n+1$ edges $e_{0}, e_{1}, \ldots, e_{n}$ and a self-adjoint matching condition at the central vertex $v$ is established. The first step is a reduction to the problem on the single edge $e_{0}$ but with an energy depending boundary condition at $v$. In the second step, by means of an abstract inverse result for $Q$-functions, a reduction to a problem on a path graph with two edges $e_{0}, \widetilde{e}_{1}$ joined by continuity and Kirchhoff conditions is given. All results are proved for symmetric linear relations in an orthogonal sum of Hilbert spaces. This ensures wide applicability to various different realizations, in particular, to canonical systems and Krein strings which include, as special cases, Dirac systems and Stieltjes strings. Employing two other key inverse results by de Branges and Krein, we answer e.g. the following question: If all differential operators are of one type, when can the reduced system be chosen to consist of two differential operators of the same type?
\end{abstract}

Communicated by Daniel Aron Alpay.

To our valued colleague Harry Dym on the occasion of his 80th birthday.

$凶 \quad$ Heinz Langer

heinz.langer@tuwien.ac.at

B. Malcolm Brown

malcolm.brown@cs.cardiff.ac.uk

Christiane Tretter

tretter@math.unibe.ch

1 School of Computer Science, Cardiff University, 5 The Parade, Cardiff CF24 3AA, UK

2 Institut für Analysis und Sci. Comp., TU Wien, Wiedner Hauptstr. 8-10, 1040 Wien, Austria

3 Mathematisches Institut, Universität Bern, Sidlerstr. 5, 3012 Bern, Switzerland

Birkhäuser 
Keywords Compressed resolvent - Differential operator - Star graph · Path graph • Canonical system $\cdot$ Krein string $\cdot$ Stieltjes string

Mathematics Subject Classification 47A10 $\cdot 34$ B45 $\cdot$ 34L40

\section{Introduction}

Direct and inverse spectral problems for differential operators on graphs have attracted rapidly increasing interest over the last two decades. Since it seems impossible to list all relevant literature, we mention the three monographs [5,26,33], whose bibliographies reflect the intense research activities in this area very well. The vast majority of these works considers concrete operators on graphs such as Sturm-Liouville and Schrödinger operators [2,3,16,25,37], Laplace and related operators [29], Krein strings [13], Stieltjes strings [30,31], canonical systems and Dirac operators [1,8]. More abstract methods were developed in $[32,35]$.

The results of this paper are of more conceptual character; they have implications to many differential and difference operators, and corresponding Cauchy problems. We establish abstract results for compressed resolvents of self-adjoint extensions of an orthogonal sum of symmetric relations. They allow us to reduce spectral problems on a star graph with self-adjoint matching condition at the central vertex to problems on a single edge and, using inverse results, to problems on a path graph with two edges, see Fig. 1a-c. We also address the question whether for concrete realizations the reduced system on the path graph can be chosen of the same type as the original system.

More precisely, the subsystem on the $n$ edges $e_{1}, e_{2}, \ldots, e_{n}$ (the blue/ non-bold part in Fig. 1a) joined to $e_{0}$ (the red/bold part in Fig. 1a) with arbitrary self-adjoint matching condition at the central vertex $v$ is first condensed into an energy depending boundary condition at the endpoint $v$ of the edge $e_{0}$ (the blue/non-bold part in Fig. 1b). In the second step the energy depending boundary condition is replaced by a new subsystem on an edge $\widetilde{e}_{1}$ joined to $e_{0}$ by continuity and Kirchhoff conditions (the blue/non-bold part in Fig. 1c).

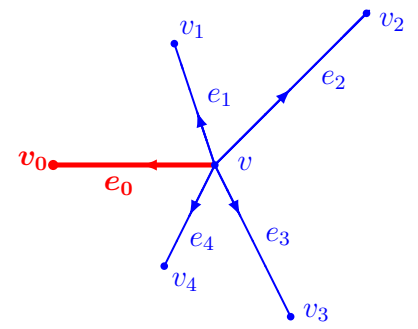

a Star graph $\mathcal{G}$ with distinguished edge $e_{0}$

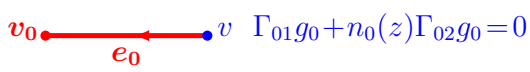

b Edge $e_{0}$ with $z$-depending 'boundary condition' at $v$

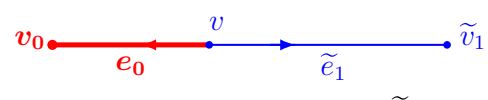

c Reduced path graph $\widetilde{\mathcal{G}}$

Fig. 1 The two-step reduction of a star graph to a path graph (color figure online) 
To exemplify our results, we consider a star graph $\mathcal{G}$ as in Fig. 1a and we suppose that for each edge $e_{j}$ a symmetric linear relation $T_{j}$ with equal defect numbers 1 in a Hilbert space $\mathcal{H}_{j}$ and a boundary triplet $\left(\mathbb{C}, \Gamma_{j 1}, \Gamma_{j 2}\right), j=0,1, \ldots, n$. are given. For concrete realizations, e.g. differential or difference operators as considered below, the boundary triplets depend on the values and derivatives of the functions at the central vertex $v$. The Hilbert space $\mathcal{H}$ on the graph $\mathcal{G}$ is the orthogonal sum of the Hilbert spaces $\mathcal{H}_{j}$ on the edges $e_{j}, j=0,1, \ldots, n$, and in $\mathcal{H}$ the symmetric relation $\mathbf{T}$ is defined as the orthogonal sum of the relations $T_{j}$. The self-adjoint extensions of $\mathbf{T}$ in $\mathcal{H}$ are all described by an interface condition parametrized by two $(n+1) \times(n+1)$ matrices $\mathcal{A}, \mathcal{B}$ (see (2.12), (2.13) below); we denote them by $\mathbf{T}_{\mathcal{A}, \mathcal{B}}$ and we fix such an extension.

The key step in our analysis is to determine the compressed resolvent of $\mathbf{T}_{\mathcal{A}, \mathcal{B}}$ corresponding to the distinguished edge $e_{0}$. This means, given an element $f_{0} \in \mathcal{H}_{0}$ we describe the first component $g_{0} \in \mathcal{H}_{0}$ of the resolvent $\left(\mathbf{T}_{\mathcal{A}, \mathcal{B}}-z\right)^{-1}$ applied to the element $\mathbf{f}_{0}=\left(f_{0}, 0, \ldots, 0\right)^{\mathrm{t}}$ supported only on $\mathcal{H}_{0}$,

$$
g_{0}=\left.P_{0}\left(\mathbf{T}_{\mathcal{A}, \mathcal{B}}-z\right)^{-1}\right|_{\mathcal{H}_{0}} f_{0}, \quad z \in \mathbb{C} \backslash \mathbb{R}
$$

where $P_{0}$ is the projection of $\mathcal{H}$ onto the first component $\mathcal{H}_{0}$. Note that the compressed resolvent of $\mathbf{T}_{\mathcal{A}, \mathcal{B}}$ (to $\mathcal{H}_{0}$ ) on the right hand side of (1.1) is also a generalized resolvent of the symmetric relation $T_{0}$ in $\mathcal{H}_{0}$. Our first main result, Theorem 3.1, shows that $g_{0}$ is a solution of the inhomogeneous problem for $T_{0}^{*}$ in $\mathcal{H}_{0}$ with energy depending boundary condition which, if $T_{0}^{*}$ is an operator, has the form

$$
\left(T_{0}^{*}-z\right) g_{0}=f_{0}, \quad \Gamma_{01} g_{0}-n_{0}(z) \Gamma_{02} g_{0}=0
$$

here the function $n_{0}$ depends on the matrices $\mathcal{A}, \mathcal{B}$ and on the Titchmarsh-Weyl functions $m_{j}$ of the other $T_{j}, j=1,2, \ldots, n$. In other words, the element $g_{0}$ in (1.1) defined by the resolvent of $\mathbf{T}_{\mathcal{A}, \mathcal{B}}$ in the Hilbert space $\mathcal{H}=\mathcal{H}_{0} \oplus \mathcal{H}_{1} \oplus \cdots \oplus \mathcal{H}_{n}$ is a solution of a problem in the first component $\mathcal{H}_{0}$, or in the graph situation on the edge $e_{0}$, but with a $z$-depending boundary condition at $v$, see Fig. $1 \mathrm{~b}$. The essential property of the function $n_{0}$ in (1.2) is that it is a Nevanlinna function, i.e. holomorphic at least on $\mathbb{C}^{+} \cup \mathbb{C}^{-}$with $n_{0}(\bar{z})=\overline{n_{0}(z)}$ and $\operatorname{Im} n_{0}(z) \geq 0$ if $\operatorname{Im} z>0$.

According to a general abstract inverse result of Langer and Textorius [28] (see also [11]), each Nevanlinna function is the $Q$-function, or Titchmarsh-Weyl function, of a symmetric relation with defect numbers 1 (and a self-adjoint extension $\widetilde{T}_{1}$ of it) in some Hilbert space $\widetilde{\mathcal{H}}_{1}$. If we apply this result to the function $n_{0}$ in (1.2), it follows that the compressed resolvent of $\mathbf{T}_{\mathcal{A}, \mathcal{B}}$ in (1.1) coincides with the compressed resolvent of a self-adjoint relation $\widetilde{\mathbf{T}}_{\mathcal{A}_{0}, \mathcal{B}_{0}}$ extending $T_{0} \oplus \widetilde{T}_{1}$ and acting in the Hilbert space $\widetilde{\mathcal{H}}=\mathcal{H}_{0} \oplus \widetilde{\mathcal{H}}_{1}$

$$
\left.P_{0}\left(\mathbf{T}_{\mathcal{A}, \mathcal{B}}-z\right)^{-1}\right|_{\mathcal{H}_{0}}=\left.\widetilde{P}_{0}\left(\widetilde{\mathbf{T}}_{\mathcal{A}_{0}, \mathcal{B}_{0}}-z\right)^{-1}\right|_{\mathcal{H}_{0}}, \quad z \in \mathbb{C} \backslash \mathbb{R}
$$

here $\mathcal{A}_{0}, \mathcal{B}_{0}$ is the matrix pair defining standard matching conditions, see Theorem 4.1. In the graph situation this means that, for given $f_{0} \in \mathcal{H}_{0}$, on the common edge 
$e_{0}$ the 'values' of $\left(\mathbf{T}_{\mathcal{A}, \mathcal{B}}-z\right)^{-1}\left(f_{0} \quad 0 \cdots \cdots\right)^{\mathrm{t}}$ for the original star graph $\mathcal{G}$ and of $\left(\widetilde{\mathbf{T}}_{\mathcal{A}_{0}, \mathcal{B}_{0}}-z\right)^{-1}\left(f_{0} 0\right)^{\mathrm{t}}$ for the simpler path graph $\widetilde{\mathcal{G}}$ coincide, see Theorem 4.4 .

This result also has implications for first and second order Cauchy problems on star graphs (see e.g. [4,23]). Consider, for example, the Cauchy problem

$$
\dot{\mathbf{u}}(t)-\mathrm{i} \mathbf{T}_{\mathcal{A}, \mathcal{B}} \mathbf{u}(t)=\mathbf{f}(t), \quad t \in[0, \infty), \quad \mathbf{u}(0)=\mathbf{u}_{0},
$$

in $\mathcal{H}$, i.e. on the star graph $\mathcal{G}$ with initial value $\mathbf{u}_{0}$ and right hand side $\mathbf{f}$ supported only on $e_{0}$. Then on this edge $e_{0}$ the solution of problem (1.4) coincides with the solution $\mathbf{u}$ of the Cauchy problem for the operator $\widetilde{\mathbf{T}}_{\mathcal{A}_{0}, \mathcal{B}_{0}}$ in $\widetilde{\mathcal{H}}$, i.e. on the path graph $\widetilde{\mathcal{G}}$, with initial value $\widetilde{\mathbf{u}}_{0}$ and right hand side $\widetilde{\mathbf{f}}_{0}$ having the same first components as $\mathbf{u}_{0}$ and $\mathbf{f}$, respectively, and zero second components, see Theorem 4.4. Analogous results can be formulated for second order Cauchy problems, see Remark 4.5.

An inverse result of de Branges [7, Thm. 7] (see also [38, Thm. 1]) yields that every (scalar) Nevanlinna function is the Titchmarsh-Weyl function of a canonical system. Hence $\widetilde{T}_{1}$ can even be chosen to be generated by a trace normed canonical system on the interval $[0, \infty)=\widetilde{e}_{1}$. Thus, in the graph situation, the compressed resolvent of $\mathbf{T}_{\mathcal{A}, \mathcal{B}}$ on the star graph, see (1.1), coincides with the compressed resolvent of an operator $\widetilde{\mathbf{T}}_{\mathcal{A}_{0}, \mathcal{B}_{0}}$ on a path graph with two edges $e_{0}, \widetilde{e}_{1}$, given by $T_{0}$ on $e_{0}$ and a canonical system on $\widetilde{e}_{1}$ joined by continuity and Kirchhoff conditions at $v$, see Theorem 4.6. If, in particular, $T_{0}$ is given by a canonical system while all other relations $T_{j}, j=1,2, \ldots, \underset{\sim}{\sim}$, are arbitrary, then $\widetilde{\mathbf{T}}_{\mathcal{A}_{0}, \mathcal{B}_{0}}$ is given by two canonical systems on the path graph $\widetilde{\mathcal{G}}$, see Corollary 5.1.

According to an inverse result of Krein [18] (see also [12, Sect. 5]), a Nevanlinna function that belongs to the subclass $\mathcal{S}$ of Stieltjes functions, i.e. is also holomorphic on $(-\infty, 0)$ and non-negative there, is the Titchmarsh-Weyl function of a Krein string. Hence, if the function $n_{0}$ in (1.2) is a Stieltjes function, the relation $\widetilde{T}_{1}$ can be chosen to be generated by a Krein string. In the case where the relations $T_{j}, j=1,2, \ldots, n$, are generated by Krein strings joined to $T_{0}$ by continuity and Robin type interface conditions at $v$, a sufficient condition for $n_{0} \in \mathcal{S}$ is that the Robin parameter $\tau$ satisfies the inequality

$$
\tau \geq-\sum_{j=1}^{n} \frac{1}{m_{j}(0-)},
$$

see Theorem 5.2. Here $m_{j}, j=1,2, \ldots, n$, is the Titchmarsh-Weyl function of the Krein string on $e_{j}$ which implies that $m_{j} \in \mathcal{S}$ and hence $m_{j}(0-) \in[0, \infty]$. Note that, for the special case of Kirchhoff conditions where $\tau=0$, condition (1.5) is automatically satisfied. Therefore, in contrast to canonical systems, if all the relations $T_{j}, j=0,1, \ldots, n$, are given by Krein strings, then $\widetilde{\mathbf{T}}_{\mathcal{A}_{0}, \mathcal{B}_{0}}$ need not be given by two Krein strings on $\widetilde{\mathcal{G}}$ unless the Robin parameter satisfies inequality (1.5).

While the mass distribution function of a Krein string is an arbitrary non-decreasing (left-continuous) function, for a Stieltjes string it is a step function with steps accumulating at most at the right end-point. For a star graph of Stieltjes strings as in Fig. 1a this means that the steps on the edges $e_{j}$ accumulate at most at the outer vertices $v_{j}$, $j=0,1, \ldots, n$. Employing asymptotic expansions of Titchmarsh-Weyl functions, it follows that if, in the above situation, inequality (1.5) holds and all $T_{j}, j=0,1, \ldots, n$, 
are Stieltjes strings, the reduced system $\widetilde{\mathbf{T}}_{\mathcal{A}_{0}, \mathcal{B}_{0}}$ consists of two Stieltjes strings, see Corollary 5.4 .

If all $T_{j}, j=0,1, \ldots, n$, are Sturm-Liouville operators, Theorem 3.1 yields a reduction to the single Sturm-Liouville operator $T_{0}$ on $e_{0}$ with an energy depending boundary condition at $v$. Moreover, Theorem 4.6 based on de Branges' inverse result applies and yields a reduction to a problem on a path graph of two edges for the SturmLiouville operator $T_{0}$ and a canonical system $\widetilde{T}_{1}$ with standard interface conditions. However, due to a lack of corresponding inverse results for Sturm-Liouville operators, the latter can in general not be replaced by a Sturm-Liouville operator.

The paper is organized as follows. In Sect. 2 we present some general results on Krein's formula for an orthogonal sum of $n+1$ symmetric linear relations $T_{j}$, $j=0,1, \ldots, n$, each with equal defect numbers 1 . In Sect. 3 we establish the relation between the compressed resolvent (1.1) and the extension of $T_{0}$ determined by an energy depending boundary condition, see (1.2). Section 4 contains the main result where we use the abstract inverse result on $Q$-functions to 'replace' the $z$-depending boundary condition by a new symmetric linear relation $\widetilde{T}_{1}$ or, correspondingly, by attaching a realization on a new edge $\widetilde{e}_{1}$, see Fig. 1c. Implications of our reduction result for Cauchy problems of first and second order on star graphs are given in Sect. 4.2. In Sect. 5 we consider concrete operators on star graphs: canonical systems and Krein strings, which cover Dirac operators and Stieltjes strings as special cases, respectively, and Sturm-Liouville operators. Combining our results with the inverse results of de Branges and Krein, we obtain a more complete picture of the structure of the reduced problems.

\section{The Abstract Schema}

In this section we set up a framework for pasting a finite number of symmetric linear relations with general self-adjoint interface conditions, comp. [35]. For this we need some notation and basic properties of symmetric linear relations, boundary triplets and corresponding Titchmarsh-Weyl functions, see e.g. [34, Chapter 14], [9,10].

\subsection{The Titchmarsh-Weyl Function of an 'Edge'}

Let $T_{0}, T_{1}, \ldots, T_{n}$ be symmetric linear relations in Hilbert spaces $\mathcal{H}_{0}, \mathcal{H}_{1}, \ldots, \mathcal{H}_{n}$ with equal defect numbers 1 . For each $j \in\{0,1, \ldots, n\}$ let $\left(\mathbb{C}, \Gamma_{j 1}, \Gamma_{j 2}\right)$ be a boundary triplet for the adjoint relation $T_{j}^{*}$, i.e.

$$
\begin{aligned}
\left(\widehat{f}_{j}, g_{j}\right)-\left(f_{j}, \widehat{g}_{j}\right) & =\left(\Gamma_{j 2} f_{j}\right) \overline{\left(\Gamma_{j 1} g_{j}\right)}-\left(\Gamma_{j 1} f_{j}\right) \overline{\left(\Gamma_{j 2} g_{j}\right)} \\
& =\left(\left(\begin{array}{cc}
0 & 1 \\
-1 & 0
\end{array}\right)\left(\begin{array}{l}
\Gamma_{j 1} f_{j} \\
\Gamma_{j 2} f_{j}
\end{array}\right),\left(\begin{array}{l}
\Gamma_{j 1} g_{j} \\
\Gamma_{j 2} g_{j}
\end{array}\right)\right), \quad\left\{f_{j}, \widehat{f}_{j}\right\},\left\{g_{j}, \widehat{g}_{j}\right\} \in T_{j}^{*}
\end{aligned}
$$


Then all self-adjoint extensions of $T_{j}$ in $\mathcal{H}_{j}$ are described by 'boundary' conditions

$$
a \Gamma_{j 1} f_{j}+b \Gamma_{j 2} f_{j}=0, \quad f_{j} \in \operatorname{dom} T_{j}^{*}
$$

with $a, b \in \mathbb{R}$. By $T_{j ; a, b}$ we denote the corresponding self-adjoint relation in $\mathcal{H}_{j}$, i.e. the restriction of $T_{j}^{*}$ by (2.2).

Choose an $\mathcal{H}_{j}$-valued function $h_{j}$ on $\mathbb{C} \backslash \mathbb{R}$ such that $h_{j}(z)$ is a defect element of $T_{j}$ at $\bar{z}$, i.e. $h_{j}(z) \in \mathcal{H}_{j} \backslash\{0\}$ and

$$
\left\{h_{j}(z), z h_{j}(z)\right\} \in T_{j}^{*}, \quad z \in \mathbb{C} \backslash \mathbb{R},
$$

and such that $h_{j}$ is analytic on $\mathbb{C} \backslash \mathbb{R}$. In fact, if we fix $z_{0} \in \mathbb{C} \backslash \mathbb{R}$ and a defect element $h_{j}\left(z_{0}\right)$ of $T_{j}$ at $\bar{z}_{0}$, i.e. $\left\{h_{j}\left(z_{0}\right), z_{0} h_{j}\left(z_{0}\right)\right\} \in T_{j}^{*}$, and, with the self-adjoint extension $T_{j ; 0,1}$ of $T_{j}$ in $\mathcal{H}_{j}$, we define

$$
h_{j}(z):=\left(T_{j ; 0,1}-z_{0}\right)\left(T_{j ; 0,1}-z\right)^{-1} h_{j}\left(z_{0}\right), \quad z \in \mathbb{C} \backslash \mathbb{R}
$$

it is easy to check that $h_{j}$ satisfies (2.3) and is analytic on $\mathbb{C} \backslash \mathbb{R}$. Further,

$$
\Gamma_{j 2} h_{j}(z)=\Gamma_{j 2}\left(h_{j}\left(z_{0}\right)+\left(z-z_{0}\right)\left(T_{j ; 0,1}-z\right)^{-1} h_{j}\left(z_{0}\right)\right)=\Gamma_{j 2} h_{j}\left(z_{0}\right), \quad z \in \mathbb{C} \backslash \mathbb{R} .
$$

In the following we choose $h_{j}\left(z_{0}\right)$ such that $\Gamma_{j 2} h_{j}\left(z_{0}\right)=1$, and hence

$$
\Gamma_{j 2} h_{j}(z)=1, \quad z \in \mathbb{C} \backslash \mathbb{R} .
$$

Then the Titchmarsh-Weyl function $m_{j}$, corresponding to this choice of the boundary triplet, is defined by the formula

$$
\Gamma_{j 1} h_{j}(z)+m_{j}(z) \Gamma_{j 2} h_{j}(z)=0, \quad z \in \mathbb{C} \backslash \mathbb{R},
$$

or equivalently, due to (2.5),

$$
m_{j}(z)=-\Gamma_{j 1} h_{j}(z), \quad z \in \mathbb{C} \backslash \mathbb{R} .
$$

The function $m_{j}$ is a Nevanlinna function since by (2.5), (2.6),

$$
\begin{aligned}
(z-\bar{\zeta})\left(h_{j}(z), h_{j}(\zeta)\right) & =\left(\widehat{h}_{j}(z), h_{j}(\zeta)\right)-\left(h_{j}(z), \widehat{h}_{j}(\zeta)\right) \\
& =\Gamma_{j 2} h_{j}(z) \overline{\Gamma_{j 1} h_{j}(\zeta)}-\Gamma_{j 1} h_{j}(z) \overline{\Gamma_{j 2} h_{j}(\zeta)} \\
& =\left(m_{j}(z)-\overline{m_{j}(\zeta)}\right) \Gamma_{j 2} h_{j}(z) \overline{\Gamma_{j 2} h_{j}(\zeta)} \\
& =m_{j}(z)-\overline{m_{j}(\zeta)}
\end{aligned}
$$


for $z, \zeta \in \mathbb{C} \backslash \mathbb{R}, z \neq \bar{\zeta}$, and hence

$$
\frac{m_{j}(z)-\overline{m_{j}(\zeta)}}{z-\bar{\zeta}}=\left(h_{j}(z), h_{j}(\zeta)\right)
$$

in particular, $\operatorname{Im} m_{j}(z)>0$ if $\operatorname{Im} z>0$.

\subsection{The 'Interface' Condition}

Now we consider the orthogonal sum $\mathbf{T}:=\bigoplus_{j=0}^{n} T_{j}$ of linear relations $T_{j}$ in the Hilbert space $\mathcal{H}:=\bigoplus_{j=0}^{n} \mathcal{H}_{j}$ with elements

$$
\mathbf{f}:=\left(f_{0} f_{1} \cdots f_{n}\right)^{\mathrm{t}}, \quad f_{j} \in \mathcal{H}_{j}, j=0,1, \ldots, n \text {. }
$$

Then $\mathbf{T}$ has equal defect numbers $n+1$ and a boundary triplet for $\mathbf{T}^{*}=\bigoplus_{j=0}^{n} T_{j}^{*}$ is given by $\left(\mathbb{C}^{n+1}, \Gamma_{1}, \Gamma_{2}\right)$ where

$$
\Gamma_{i} \mathbf{f}:=\left(\Gamma_{0 i} f_{0} \Gamma_{1 i} f_{1} \cdots \Gamma_{n i} f_{n}\right)^{\mathrm{t}}, \quad \mathbf{f} \in \mathcal{H}, \quad i=1,2,
$$

since the relations (2.1) imply that

$$
\begin{aligned}
(\widehat{\mathbf{f}}, \mathbf{g})-(\mathbf{f}, \widehat{\mathbf{g}}) & =\left(\Gamma_{2} \mathbf{f}, \Gamma_{1} \mathbf{g}\right)_{\mathbb{C}^{n+1}}-\left(\Gamma_{1} \mathbf{f}, \Gamma_{2} \mathbf{g}\right)_{\mathbb{C}^{n+1}} \\
& \left.=\left(\begin{array}{cc}
0 & \mathcal{I}_{n} \\
-\mathcal{I}_{n} & 0
\end{array}\right)\left(\begin{array}{l}
\Gamma_{1} \mathbf{f} \\
\Gamma_{2} \mathbf{f}
\end{array}\right), \quad\left(\begin{array}{l}
\Gamma_{1} \mathbf{g} \\
\Gamma_{2} \mathbf{g}
\end{array}\right)\right)_{\mathbb{C}^{2(n+1)}}, \quad\left\{\mathbf{f}, \widehat{\mathbf{f}\}}, \quad\{\mathbf{g}, \widehat{\mathbf{g}}\} \in \mathbf{T}^{*}\right.
\end{aligned}
$$

If we define the $\mathcal{H}$-valued function $\mathbf{h}$ by

$$
\mathbf{h}(z):=\left(h_{0}(z) h_{1}(z) \cdots h_{n}(z)\right)^{\mathrm{t}}, \quad z \in \mathbb{C} \backslash \mathbb{R},
$$

then $\{\mathbf{h}(z), z \mathbf{h}(z)\} \in \mathbf{T}^{*}$ and the corresponding Titchmarsh-Weyl function is the $(n+1) \times(n+1)$-matrix function $\mathcal{M}$ defined by

$$
\Gamma_{1} \mathbf{h}(z)+\mathcal{M}(z) \Gamma_{2} \mathbf{h}(z)=0, \quad z \in \mathbb{C} \backslash \mathbb{R}
$$

Clearly, due to (2.6),

$$
\mathcal{M}(z)=\operatorname{diag}\left(m_{0}(z), m_{1}(z), \ldots, m_{n}(z)\right), \quad z \in \mathbb{C} \backslash \mathbb{R}
$$

and hence, using (2.5) we conclude that

$$
\operatorname{diag}\left(\Gamma_{01} h_{0}(z), \ldots, \Gamma_{n 1} h_{n}(z)\right)+\mathcal{M}(z)=0, \quad z \in \mathbb{C} \backslash \mathbb{R}
$$

A description of all self-adjoint extensions of symmetric linear relations with equal deficiency numbers in terms of a boundary triplet has long been known (see 
[6,22], [10]). Correspondingly, all self-adjoint extensions of $\mathbf{T}$ in $\mathcal{H}$ are given by 'interface' conditions

$$
\mathcal{A} \Gamma_{1} \mathbf{y}+\mathcal{B} \Gamma_{2} \mathbf{y}=0
$$

where $\mathcal{A}, \mathcal{B}$ is a pair of real $(n+1) \times(n+1)$-matrices with the properties

$$
\operatorname{rank}(\mathcal{A} \mathcal{B})=n+1, \quad \mathcal{A B}^{*}=\mathcal{B} \mathcal{A}^{*}
$$

By $\mathbf{T}_{\mathcal{A}, \mathcal{B}}$ we denote the corresponding self-adjoint relation in $\mathcal{H}$, i.e. the restriction of $\mathbf{T}^{*}$ by (2.12).

It is well-known that the inverse $(\mathcal{A M}(z)-\mathcal{B})^{-1}$ exists for all $z \in \mathbb{C} \backslash \mathbb{R}$. In fact, the Nevanlinna property of $\mathcal{M}$ and (2.13) imply that, for $x_{0} \in \operatorname{ker}\left(\mathcal{M}(z)^{*} \mathcal{A}^{*}-\mathcal{B}^{*}\right)$,

$$
0=\operatorname{Im}\left(\left(\mathcal{M}(z)^{*} \mathcal{A}^{*}-\mathcal{B}^{*}\right) x_{0}, \mathcal{A}^{*} x_{0}\right)_{\mathbb{C}^{n+1}}=\operatorname{Im}\left(\mathcal{M}(z)^{*} \mathcal{A}^{*} x_{0}, \mathcal{A}^{*} x_{0}\right)_{\mathbb{C}^{n+1}},
$$

whence $\mathcal{A}^{*} x_{0}=0$ and thus $\mathcal{B}^{*} x_{0}=0$, a contradiction to the first condition in (2.13).

\subsection{A Version of M. G. Krein's Resolvent Formula}

The resolvent of any self-adjoint relation $\mathbf{T}_{\mathcal{A}, \mathcal{B}}$ is related to the resolvent of the particular self-adjoint relation $\mathbf{T}_{0, \mathcal{I}}$ with the special 'interface' condition $\Gamma_{2} \mathbf{y}=0$ as follows.

Proposition 2.1 For $z \in \mathbb{C} \backslash \mathbb{R}$ and $\mathbf{f} \in \mathcal{H}$, we have

$$
\begin{aligned}
& \left(\mathbf{T}_{\mathcal{A}, \mathcal{B}}-z\right)^{-1} \mathbf{f}=\left(\mathbf{T}_{0, \mathcal{I}}-z\right)^{-1} \mathbf{f} \\
& -\operatorname{diag}\left(h_{0}(z), h_{1}(z), \ldots, h_{n}(z)\right)(\mathcal{A M}(z)-\mathcal{B})^{-1} \mathcal{A}\left(\begin{array}{c}
\left(f_{0}, h_{0}(\bar{z})\right)\left(f_{1}, h_{1}(\bar{z})\right) \\
\vdots \\
\left(f_{n}, h_{n}(\bar{z})\right)
\end{array}\right),
\end{aligned}
$$

where $h_{j}(z)$ and $\mathcal{M}(z)$ are as in (2.4) and (2.10), respectively.

Proof Let $z \in \mathbb{C} \backslash \mathbb{R}$. For given $\mathbf{f} \in \mathcal{H}$ the element $\mathbf{y}:=\left(\mathbf{T}_{\mathcal{A}, \mathcal{B}}-z\right)^{-1} \mathbf{f}$ given by

$$
\{\mathbf{y}(z), \widehat{\mathbf{y}}(z)\} \in \mathbf{T}_{\mathcal{A}, \mathcal{B}}, \quad \widehat{\mathbf{y}}(z)-z \mathbf{y}(z)=\mathbf{f} .
$$

In the following, for ease of presentation, we restrict ourselves to the case that all $T_{j}, j=0,1, \ldots, n$, are operators, and hence so are $\mathbf{T}_{\mathcal{A}, \mathcal{B}}$ and $\mathbf{T}_{0, \mathcal{I}}$. We consider the difference of the resolvents of $\mathbf{T}_{\mathcal{A}, \mathcal{B}}$ and $\mathbf{T}_{0, \mathcal{I}}$. Recall that, due to (2.2) and (2.12),

$$
\Gamma_{j 2} \operatorname{dom} T_{j ; 0,1}=\{0\}, \quad \Gamma_{2} \operatorname{dom} \mathbf{T}_{0, \mathcal{I}}=\{0\} .
$$


Then, for $\mathbf{f} \in \mathcal{H}$, we have

$$
\left(\mathbf{T}^{*}-z\right)\left(\left(\mathbf{T}_{\mathcal{A}, \mathcal{B}}-z\right)^{-1} \mathbf{f}-\left(\mathbf{T}_{0, \mathcal{I}}-z\right)^{-1} \mathbf{f}\right)=0
$$

and hence, by (2.3),

$$
\begin{aligned}
\left(\mathbf{T}_{\mathcal{A}, \mathcal{B}}-z\right)^{-1} \mathbf{f} & -\left(\mathbf{T}_{0, \mathcal{I}}-z\right)^{-1} \mathbf{f} \\
& =\left(c_{0}(\mathbf{f} ; z) h_{0}(z) c_{1}(\mathbf{f} ; z) h_{1}(z) \cdots c_{n}(\mathbf{f} ; z) h_{n}(z)\right)^{\mathrm{t}}
\end{aligned}
$$

with linear functionals $c_{j}(\cdot, z): \mathcal{H} \rightarrow \mathbb{C}, j=0,1, \ldots, n$. Then, for $\mathbf{f}=\left(f_{j}\right)_{0}^{n}$, $\mathbf{g}=\left(g_{j}\right)_{0}^{n} \in \mathcal{H}$, taking the scalar product with $\mathbf{g}$ in (2.16), we conclude that

$$
\begin{aligned}
\left(\left(\mathbf{T}_{\mathcal{A}, \mathcal{B}}-z\right)^{-1} \mathbf{f}, \mathbf{g}\right) & -\left(\left(\mathbf{T}_{0, \mathcal{I}}-z\right)^{-1} \mathbf{f}, \mathbf{g}\right) \\
& =\left(c_{0}(\mathbf{f} ; z) c_{1}(\mathbf{f} ; z) \cdots c_{n}(\mathbf{f} ; z)\right)\left(\begin{array}{c}
\left(h_{0}(z), g_{0}\right) \\
\left(h_{1}(z), g_{1}\right) \\
\vdots \\
\left(h_{n}(z), g_{n}\right)
\end{array}\right)
\end{aligned}
$$

and, analogously,

$$
\begin{aligned}
\overline{\left(\left(\mathbf{T}_{\mathcal{A}, \mathcal{B}}-\bar{z}\right)^{-1} \mathbf{g}, \mathbf{f}\right)}-\overline{\left(\left(\mathbf{T}_{0, \mathcal{I}}-\bar{z}\right)^{-1} \mathbf{g}, \mathbf{f}\right)} \\
=\left(\overline{c_{0}(\mathbf{g} ; \bar{z})} \overline{c_{1}(\mathbf{g} ; \bar{z})} \cdots \overline{c_{n}(\mathbf{g} ; \bar{z})}\right)\left(\begin{array}{c}
\left(f_{0}, h_{0}(\bar{z})\right) \\
\left(f_{1}, h_{1}(\bar{z})\right) \\
\vdots \\
\left(f_{n}, h_{n}(\bar{z})\right)
\end{array}\right) .
\end{aligned}
$$

Since $\mathbf{T}_{\mathcal{A}, \mathcal{B}}$ and $\mathbf{T}_{0, \mathcal{I}}$ are self-adjoint, it follows that the two right-hand sides above are equal. It is not difficult to see that this implies that there exists an $(n+1) \times(n+1)$-matrix $\mathcal{F}(z)$ such that

$$
\begin{aligned}
\left(\mathbf{T}_{\mathcal{A}, \mathcal{B}}-z\right)^{-1} \mathbf{f}=\left(\mathbf{T}_{0, \mathcal{I}}-z\right)^{-1} \mathbf{f} \\
\\
+\operatorname{diag}\left(h_{0}(z), h_{1}(z), \ldots, h_{n}(z)\right) \mathcal{F}(z)\left(\begin{array}{c}
\left(f_{0}, h_{0}(\bar{z})\right) \\
\left(f_{1}, h_{1}(\bar{z})\right) \\
\vdots \\
\left(f_{n}, h_{n}(\bar{z})\right)
\end{array}\right)
\end{aligned}
$$


In order to find an expression for $\mathcal{F}(z)$, we apply the functionals $\Gamma_{1}$ and $\Gamma_{2}$ to (2.17) and observe (2.11), (2.5), and (2.15) to obtain

$$
\begin{aligned}
& \Gamma_{1}\left(\mathbf{T}_{\mathcal{A}, \mathcal{B}}-z\right)^{-1} \mathbf{f}=\Gamma_{1}\left(\mathbf{T}_{0, \mathcal{I}}-z\right)^{-1} \mathbf{f}-\mathcal{M}(z) \mathcal{F}(z)\left(\begin{array}{c}
\left(f_{0}, h_{0}(\bar{z})\right) \\
\left(f_{1}, h_{1}(\bar{z})\right) \\
\vdots \\
\left(f_{n}, h_{n}(\bar{z})\right)
\end{array}\right), \\
& \Gamma_{2}\left(\mathbf{T}_{\mathcal{A}, \mathcal{B}}-z\right)^{-1} \mathbf{f}=\mathcal{F}(z)\left(\begin{array}{c}
\left(f_{0}, h_{0}(\bar{z})\right) \\
\left(f_{1}, h_{1}(\bar{z})\right) \\
\vdots \\
\left(f_{n}, h_{n}(\bar{z})\right)
\end{array}\right) .
\end{aligned}
$$

By means of the relation (2.12) for $\mathbf{y}=\left(\mathbf{T}_{\mathcal{A}, \mathcal{B}}-z\right)^{-1} \mathbf{f}$, we arrive at

$$
\mathcal{A} \Gamma_{1}\left(\mathbf{T}_{0, \mathcal{I}}-z\right)^{-1} \mathbf{f}-(\mathcal{A M}(z)-\mathcal{B}) \mathcal{F}(z)\left(\begin{array}{c}
\left(f_{0}, h_{0}(\bar{z})\right) \\
\left(f_{1}, h_{1}(\bar{z})\right) \\
\vdots \\
\left(f_{n}, h_{n}(\bar{z})\right)
\end{array}\right)=0 .
$$

Further, (2.1) yields that

$$
\begin{aligned}
& \left(T_{j}^{*}\left(T_{j ; 0,1}-z\right)^{-1} f_{j}, h_{j}(\bar{z})\right)-\left(\left(T_{j ; 0,1}-z\right)^{-1} f_{j}, T_{j}^{*} h_{j}(\bar{z})\right) \\
& \quad=\left(\Gamma_{j 2}\left(T_{j ; 0,1}-z\right)^{-1} f_{j}\right) \overline{\Gamma_{j 1} h_{j}(\bar{z})}-\left(\Gamma_{j 1}\left(T_{j: 0,1}-z\right)^{-1} f_{j}\right) \overline{\Gamma_{j 2} h_{j}(\bar{z})} .
\end{aligned}
$$

Since $T_{j}^{*} h_{j}(\bar{z})=\bar{z} h_{j}(\bar{z})$ by (2.3), the expression on the left hand side equals $\left(f_{j}, h_{j}(\bar{z})\right)$. The first term on the right hand side is zero because the first factor vanishes, and the second factor of the last term is equal to 1 due to (2.5). It follows that

$$
\left(f_{j}, h_{j}(\bar{z})\right)=-\Gamma_{j 1}\left(T_{j ; 0,1}-z\right)^{-1} f_{j}, \quad f_{j} \in \mathcal{H}_{j}, j=0,1, \ldots, n
$$

Using this in (2.18), we find

$$
\mathcal{F}(z)=-(\mathcal{A M}(z)-\mathcal{B})^{-1} \mathcal{A}
$$

Inserting this expression into (2.17) we arrive at (2.14).

\section{The Compressed Resolvent}

In this section we characterize the compression of the resolvent of $\mathbf{T}_{\mathcal{A}, \mathcal{B}}$ to the first component $\mathcal{H}_{0}$ of $\mathcal{H}$,

$$
\left.P_{0}\left(\mathbf{T}_{\mathcal{A}, \mathcal{B}}-z\right)^{-1}\right|_{\mathcal{H}_{0}}=P_{0}\left(\mathbf{T}_{\mathcal{A}, \mathcal{B}}-z\right)^{-1} P_{0}^{*},
$$


in terms of $T_{0}^{*}$ and a $z$-depending boundary condition. Here $P_{0}$ is the projection onto the first component, $P_{0}: \mathcal{H} \rightarrow \mathcal{H}_{0}, P_{0}\left(f_{j}\right)_{0}^{n}=f_{0}$, and $P_{0}^{*}: \mathcal{H}_{0} \rightarrow \mathcal{H}$, $P_{0}^{*} f_{0}=\left(\begin{array}{llll}f_{0} & 0 & \cdots & 0\end{array}\right)^{\mathrm{t}}$.

Theorem 3.1 Let $\mathcal{M}_{n}=\operatorname{diag}\left(0, m_{1}, \ldots, m_{n}\right)$ where $m_{j}$ are the Titchmarsh-Weyl functions defined by (2.6), $j=0,1, \ldots, n$, and let $\mathrm{e}_{0} \in \mathbb{C}^{n+1}$ be the first unit vector. Then, for $z \in \mathbb{C} \backslash \mathbb{R}$, the problem

$$
\left.P_{0}\left(\mathbf{T}_{\mathcal{A}, \mathcal{B}}-z\right)^{-1}\right|_{\mathcal{H}_{0}} f_{0}=g_{0}, \quad f_{0}, g_{0} \in \mathcal{H}_{0},
$$

is equivalent to the inhomogeneous problem in $\mathcal{H}_{0}$ with z-depending boundary condition

$$
\left\{g_{0}, f_{0}\right\} \in\left(T_{0}^{*}-z\right), \quad \Gamma_{01} g_{0}-n_{0}(z) \Gamma_{02} g_{0}=0,
$$

where $n_{0}$ is a Nevanlinna function not depending on $m_{0}$ and given by

$$
n_{0}(z):=\frac{1}{\left(\left(\mathcal{A} \mathcal{M}_{n}(z)-\mathcal{B}\right)^{-1} \mathcal{A} \mathrm{e}_{0}, \mathrm{e}_{0}\right)_{\mathbb{C}^{n+1}}}, \quad z \in \mathbb{C} \backslash \mathbb{R},
$$

or $n_{0} \equiv \infty$ (corresponding to $\Gamma_{01} g_{0}=0$ in (3.2)).

Remark 3.2 The statement of Theorem 3.1 may also be formulated as follows. If $\mathbf{f} \in \mathcal{H}$ is supported on $\mathcal{H}_{0}$, then, for $z \in \rho\left(\mathbf{T}_{\mathcal{A}, \mathcal{B}}\right)$ the projection of $\left(\mathbf{T}_{\mathcal{A}, \mathcal{B}}-z\right)^{-1} \mathbf{f}$ to $\mathcal{H}_{0}$ is the solution of the boundary value problem for $T_{0}^{*}-z$ on $\mathcal{H}_{0}$ with the $z$-depending boundary condition in (3.2). Note that, if $T_{0}$ is a linear operator, then (3.2) becomes

$$
\left(T_{0}^{*}-z\right) g_{0}=f_{0}, \quad \Gamma_{01} g_{0}-n_{0}(z) \Gamma_{02} g_{0}=0 .
$$

Proof of Theorem 3.1 For ease of presentation, we assume that $T_{0}$ is a linear operator. Let $z \in \mathbb{C} \backslash \mathbb{R}$. First we prove that if $f_{0}, g_{0} \in \mathcal{H}_{0}$ satisfy (3.1), then they satisfy (3.2). By (3.1) and since $\mathbf{T}=\bigoplus_{j=0}^{n} T_{j} \subset \mathbf{T}_{\mathcal{A}, \mathcal{B}}$, we conclude that, for arbitrary $u_{0} \in \operatorname{dom} T_{0}$,

$$
\begin{aligned}
\left(g_{0},\left(T_{0}-\bar{z}\right) u_{0}\right) & =\left(\left.P_{0}\left(\mathbf{T}_{\mathcal{A}, \mathcal{B}}-z\right)^{-1}\right|_{\mathcal{H}_{0}} f_{0},\left(T_{0}-\bar{z}\right) u_{0}\right) \\
& =\left(f_{0},\left.P_{0}\left(\mathbf{T}_{\mathcal{A}, \mathcal{B}}-\bar{z}\right)^{-1}\right|_{\mathcal{H}_{0}}\left(T_{0}-\bar{z}\right) u_{0}\right)=\left(f_{0}, u_{0}\right) .
\end{aligned}
$$

This implies $g_{0} \in \operatorname{dom} T_{0}^{*}$ and $\left(T_{0}^{*}-z\right) g_{0}=f_{0}$, which proves the first claim in (3.2).

Further, applying $P_{0}$ to (2.14) for $\mathbf{f}=P_{0}^{*} f_{0}$ with $f_{0} \in \mathcal{H}_{0}$, we find

$$
\begin{aligned}
g_{0} & =\left.P_{0}\left(\mathbf{T}_{\mathcal{A}, \mathcal{B}}-z\right)^{-1}\right|_{\mathcal{H}_{0}} f_{0} \\
& =\left(T_{1 ; 0,1}-z\right)^{-1} f_{0}-h_{0}(z)\left((A \mathcal{M}(z)-\mathcal{B})^{-1} \mathcal{A} \mathrm{e}_{0}, \mathrm{e}_{0}\right)_{\mathbb{C}^{n+1}}\left(f_{0}, h_{0}(\bar{z})\right) .
\end{aligned}
$$

If we use (2.19) and (2.7), it follows that 
$\Gamma_{01} g_{0}$

$$
\begin{aligned}
& =\left.\Gamma_{01} P_{0}\left(\mathbf{T}_{\mathcal{A}, \mathcal{B}}-z\right)^{-1}\right|_{\mathcal{H}_{0}} f_{0} \\
& =\Gamma_{01}\left(T_{1 ; 0,1}-z\right)^{-1} f_{0}-\Gamma_{01} h_{0}(z)\left((A \mathcal{M}(z)-\mathcal{B})^{-1} \mathcal{A} \mathrm{e}_{0}, \mathrm{e}_{0}\right)_{\mathbb{C}^{n+1}}\left(f_{0}, h_{0}(\bar{z})\right) \\
& =-\left(1-m_{0}(z)\left((A \mathcal{M}(z)-\mathcal{B})^{-1} \mathcal{A e}_{0}, \mathrm{e}_{0}\right)_{\mathbb{C}^{n+1}}\right)\left(f_{0}, h_{0}(\bar{z})\right) .
\end{aligned}
$$

Similarly, observing (2.15) and (2.5), we conclude that

$$
\begin{aligned}
& \Gamma_{02} g_{0} \\
& \quad=\left.\Gamma_{02} P_{0}\left(\mathbf{T}_{\mathcal{A}, \mathcal{B}}-z\right)^{-1}\right|_{\mathcal{H}_{0}} f_{0} \\
& \quad=\Gamma_{02}\left(T_{1 ; 0,1}-z\right)^{-1} f_{0}-\Gamma_{02} h_{0}(z)\left((A \mathcal{M}(z)-\mathcal{B})^{-1} \mathcal{A e}_{0}, \mathrm{e}_{0}\right)_{\mathbb{C}^{n+1}}\left(f_{0}, h_{0}(\bar{z})\right) \\
& \quad=-\left((A \mathcal{M}(z)-\mathcal{B})^{-1} \mathcal{A e}_{0}, \mathrm{e}_{0}\right)_{\mathbb{C}^{n+1}}\left(f_{0}, h_{0}(\bar{z})\right)
\end{aligned}
$$

Altogether, we obtain

$$
\frac{\Gamma_{01} g_{0}}{\Gamma_{02} g_{0}}=\frac{1-m_{0}(z)\left((A \mathcal{M}(z)-\mathcal{B})^{-1} \mathcal{A} \mathrm{e}_{0}, \mathrm{e}_{0}\right)_{\mathbb{C}^{n+1}}}{\left((A \mathcal{M}(z)-\mathcal{B})^{-1} \mathcal{A} \mathrm{e}_{0}, \mathrm{e}_{0}\right)_{\mathbb{C}^{n+1}}}=\tilde{n}_{0}(z),
$$

and hence $g_{1}$ satisfies the boundary condition in (3.2) if we show that $\tilde{n}_{0}=n_{0}$.

Next we prove that if $f_{0}, g_{0} \in \mathcal{H}_{0}$ satisfy (3.2), then they satisfy (3.1). This means that we have to show that $\widetilde{g}_{0}:=P_{0}\left(\mathbf{T}_{\mathcal{A}, \mathcal{B}}-z\right)^{-1}\left(T_{0}^{*}-z\right) g_{0}$ is equal to $g_{0}$. The definition of $\widetilde{g}_{0}$ implies that $f_{0}$ and $\widetilde{g}_{0}$ satisfy (3.1) and hence, by what has been already proved in the first part, also (3.2), i.e.

$$
\left(T_{0}^{*}-z\right) \widetilde{g}_{0}=f_{0}, \quad \Gamma_{01} \widetilde{g}_{0}-n_{0}(z) \Gamma_{02} \widetilde{g}_{0}=0 .
$$

Thus the difference $h:=\widetilde{g}_{0}-g_{0}$ is a solution of the homogeneous problem

$$
\left(T_{0}^{*}-z\right) h=0, \quad \Gamma_{01} h-n_{0}(z) \Gamma_{02} h=0 .
$$

This together with (2.1) yields that

$$
\begin{aligned}
2 \mathrm{i}(\operatorname{Im} z)\|h\|^{2}=(z-\bar{z})\|h\|^{2} & =\left(T_{0}^{*} h, h\right)-\left(h, T_{0}^{*} h\right) \\
& =\left(\Gamma_{02} h\right) \overline{\left(\Gamma_{01} h\right)}-\left(\Gamma_{01} h\right) \overline{\left(\Gamma_{02} h\right)} \\
& =\left(\overline{n_{0}(z)}-n_{0}(z)\right)\left|\Gamma_{02} h\right|^{2} \\
& =-2 \mathrm{i}\left(\operatorname{Im} n_{0}(z)\right)\left|\Gamma_{02} h\right|^{2} .
\end{aligned}
$$

If $h \neq 0$, this would imply that $\operatorname{Im} n_{0}(z) / \operatorname{Im} z<0$, which is a contradiction if we prove that $n_{0}$ is a Nevanlinna function.

It remains to be shown that $\widetilde{n}_{0}=n_{0}$ and that $n_{0}$ is a Nevanlinna function. Since the latter clearly holds for $n_{0} \equiv 0$, we may assume that we have 
$1-m_{0}(z)\left((A \mathcal{M}(z)-\mathcal{B})^{-1} \mathcal{A} \mathrm{e}_{0}, \mathrm{e}_{0}\right)_{\mathbb{C}^{n+1}} \neq 0$. We introduce the $(n+1) \times(n+1)$ matrix function

$$
\mathcal{N}(z):=\mathcal{A M}_{n}(z)-\mathcal{B}=\mathcal{A}\left(\mathcal{M}(z)-m_{0}(z)\left(\cdot, \mathrm{e}_{0}\right)_{\mathbb{C}^{n+1}} \mathrm{e}_{0}\right)-\mathcal{B}, \quad z \in \mathbb{C} \backslash \mathbb{R}
$$

It is not difficult to show that $\mathcal{N}(z)^{-1}$ exists for $z \in \mathbb{C} \backslash \mathbb{R}$ and is given by

$$
\begin{aligned}
\mathcal{N}(z)^{-1}= & m_{0}(z) \frac{\left((A \mathcal{M}(z)-\mathcal{B})^{-1} \cdot, \mathrm{e}_{0}\right)_{\mathbb{C}^{n+1}}}{1-m_{0}(z)\left((A \mathcal{M}(z)-\mathcal{B})^{-1} \mathcal{A} \mathrm{e}_{0}, \mathrm{e}_{0}\right)_{\mathbb{C}^{n+1}}}(A \mathcal{M}(z)-\mathcal{B})^{-1} \mathcal{A} \mathrm{e}_{0} \\
& +(A \mathcal{M}(z)-\mathcal{B})^{-1}
\end{aligned}
$$

By (3.3), this implies that, for $z \in \mathbb{C} \backslash \mathbb{R}$,

$$
\frac{1}{n_{0}(z)}=\left(\mathcal{N}(z)^{-1} \mathcal{A} \mathrm{e}_{0}, \mathrm{e}_{0}\right)=\frac{\left((\mathcal{A M}(z)-\mathcal{B})^{-1} \mathcal{A} \mathrm{e}_{0}, \mathrm{e}_{0}\right)_{\mathbb{C}^{n+1}}}{1-m_{0}(z)\left((\mathcal{A M}(z)-\mathcal{B})^{-1} \mathcal{A} \mathrm{e}_{0}, \mathrm{e}_{0}\right)_{\mathbb{C}^{n+1}}}=\frac{1}{\widetilde{n}_{0}(z)}
$$

Hence $\widetilde{n}_{0}=n_{0}$ and $n_{0}$ is a Nevanlinna function if $-\mathcal{N}(z)^{-1} \mathcal{A}$ is a matrix Nevanlinna function. Since the $m_{j}$ are Nevanlinna functions and $\mathcal{B A}^{*}$ is self-adjoint, (3.4) shows that $\mathcal{N}(z) \mathcal{A}^{*}$ is a matrix Nevanlinna function and hence so is $-\mathcal{N}(z)^{-1} \mathcal{A}$ because

$$
\frac{-\mathcal{N}(z)^{-1} \mathcal{A}+\mathcal{A}^{*} \mathcal{N}(z)^{-*}}{z-\bar{z}}=\mathcal{N}(z)^{-1} \frac{-\mathcal{A N}(z)^{*}+\mathcal{N}(z) \mathcal{A}^{*}}{z-\bar{z}} \mathcal{N}(z)^{-*}, \quad z \in \mathbb{C} \backslash \mathbb{R}
$$

That $n_{0}$ is independent of $m_{0}$ is immediate from (3.3).

Theorem 3.1 may be used to relate the spectra of $\mathbf{T}_{\mathcal{A}, \mathcal{B}}$ in $\mathcal{H}$ and of the $z$-depending spectral problem (3.2) in $\mathcal{H}_{0}$. For simplicity, we consider the case that the self-adjoint extensions of the $T_{j}$ have discrete spectra, hence all the functions $m_{j}$ and also $n_{0}$ are meromorphic. Note that, if $z=\lambda$ is a pole of $n_{0}$, then the second relation in (3.2) becomes $\Gamma_{02} g=0$; if $z=\lambda$ is a zero of $n_{0}$ or if $n_{0} \equiv 0$, then the second relation in (3.2) becomes $\Gamma_{01} g=0$.

Corollary 3.3 (i) If $z \in \rho\left(\mathbf{T}_{\mathcal{A}, \mathcal{B}}\right)$, then for each $f_{0} \in \mathcal{H}_{0}$ the problem (3.2) has the solution $g_{0}$ given by (3.1).

(ii) If $\lambda \in \sigma_{\mathrm{p}}\left(\mathbf{T}_{\mathcal{A}, \mathcal{B}}\right)$ with eigenvector $\mathbf{g}$ and $n_{0}$ is holomorphic in a neighbourhood of $\lambda$, then

$$
\left\{P_{0} \mathbf{g}, 0\right\} \in\left(T_{0}^{*}-z\right), \quad \Gamma_{01} P_{0} \mathbf{g}-n_{0}(z) \Gamma_{02} P_{0} \mathbf{g}=0,
$$

is satisfied for $z=\lambda$, and $\lambda$ is an eigenvalue of (3.5) with eigenvector $P_{0} \mathbf{g}$ if $P_{0} \mathbf{g} \neq 0$.

Proof The first claim is immediate from Theorem 3.1, the second claim follows because 


$$
\begin{aligned}
\left(\left(T_{0}^{*}-\lambda\right) P_{0} \mathbf{g}, u\right) & =\left(P_{0} \mathbf{g},\left(T_{0}-\lambda\right) u\right)=\left(\mathbf{g},\left(T_{0}-\lambda\right) u\right) \\
& =\left(\mathbf{g},\left(\mathbf{T}_{\mathcal{A}, \mathcal{B}}-\lambda\right) u\right)=\left(\left(\mathbf{T}_{\mathcal{A}, \mathcal{B}}-\lambda\right) \mathbf{g}, u\right)=0
\end{aligned}
$$

for $u \in \operatorname{dom}\left(T_{0}\right)$.

Example 3.4 As a special case of interface conditions (2.12) we consider

$$
\begin{aligned}
& \Gamma_{01} f_{0}=\Gamma_{11} f_{1}=\cdots=\Gamma_{n 1} f_{n}, \\
& \Gamma_{01} f_{0}=\frac{1}{\tau}\left(\Gamma_{02} f_{0}+\Gamma_{12} f_{1}+\cdots+\Gamma_{n 2} f_{n}\right),
\end{aligned}
$$

where $\tau \in \mathbb{R} \cup\{\infty\}$. Here $\tau=0$ corresponds to continuity and Kirchhoff type conditions, while $\tau=\infty$ corresponds to Dirichlet type conditions where the relations $T_{j}$ are decoupled. If $\tau \neq 0$, the matrices $\mathcal{A}$ and $\mathcal{B}$ in (2.12) can be chosen as

$$
\mathcal{A}_{\tau}=\left(\begin{array}{cccccc}
1 & -1 & 0 & \cdots & 0 & 0 \\
0 & 1 & -1 & \cdots & 0 & 0 \\
\vdots & \vdots & \vdots & & \vdots & \vdots \\
0 & 0 & 0 & \cdots & 1 & -1 \\
1 & 0 & 0 & \cdots & 0 & 0
\end{array}\right), \mathcal{B}_{\tau}=-\frac{1}{\tau}\left(\begin{array}{cccccc}
0 & 0 & 0 & \cdots & 0 & 0 \\
0 & 0 & 0 & \cdots & 0 & 0 \\
\vdots & \vdots & \vdots & & \vdots & \vdots \\
0 & 0 & 0 & \cdots & 0 & 0 \\
1 & 1 & 1 & \cdots & 1 & 1
\end{array}\right) .
$$

For $z \in \mathbb{C} \backslash \mathbb{R}$, the solution $x(z)=\left(x_{j}(z)\right)_{0}^{n} \in \mathbb{C}^{n+1}$ of the inhomogeneous linear system $\left(\mathcal{A}_{\tau} \mathcal{M}(z)-\mathcal{B}_{\tau}\right) x(z)=\mathcal{A}_{\tau} \mathrm{e}_{0}$ is given by

$$
\begin{aligned}
& x_{j}(z)=\left(m_{0}(z) x_{0}(z)-1\right) \frac{1}{m_{j}(z)}, \quad j=1,2, \ldots, n \\
& m_{0}(z) x_{0}(z)+\frac{1}{\tau}\left(x_{0}(z)+\left(m_{0}(z) x_{0}(z)-1\right) \sum_{j=1}^{n} \frac{1}{m_{j}(z)}\right)=1,
\end{aligned}
$$

and hence we conclude that the function $n_{0}$ in (3.3) takes the form

$$
n_{0}(z)=\frac{1}{x_{0}(z)}-m_{0}(z)=\frac{1}{\tau+\frac{1}{m_{1}(z)}+\frac{1}{m_{2}(z)}+\cdots+\frac{1}{m_{n}(z)}} .
$$

It is not difficult to see that (3.8) continues to hold if $\tau=0$; note that here the matrices $\mathcal{A}_{0}, \mathcal{B}_{0}$ have to be chosen differently from (3.7), see (3.10) below for the case $n=1$.

In the special case of Robin type interface conditions, the properties of $n_{0}$ proved in Theorem 3.1 for general interface conditions (2.12) are immediate from the explicit formula (3.8) for $n_{0}: n_{0}$ does not depend on $m_{0}$, and since $m_{1}, m_{2}, \ldots, m_{n}$ are Nevanlinna functions so is $n_{0}$ for every $\tau \in \mathbb{R} \cup\{\infty\}$.

Remark 3.5 The special case $n=1$ and $\tau=0$ corresponds to the linear relation $\mathbf{T}=T_{0} \oplus T_{1}$ in $\mathcal{H}=\mathcal{H}_{0} \oplus \mathcal{H}_{1}$ and its self-adjoint extension $\mathbf{T}_{\mathcal{A}_{0}, \mathcal{B}_{0}}$ given by continuity and Kirchhoff type conditions 


$$
\Gamma_{01} f_{0}=\Gamma_{11} f_{1}, \quad \Gamma_{02} f_{0}=-\Gamma_{12} f_{1},
$$

where $\mathcal{A}, \mathcal{B}$ in (2.12) can be chosen as

$$
\mathcal{A}_{0}=\left(\begin{array}{cc}
1 & -1 \\
0 & 0
\end{array}\right), \quad \mathcal{B}_{0}=\left(\begin{array}{ll}
0 & 0 \\
1 & 1
\end{array}\right) .
$$

Here formula (3.8) reduces to

$$
n_{0}(z)=m_{1}(z), \quad z \in \mathbb{C} \backslash \mathbb{R},
$$

that is, the $z$-depending coefficient of the boundary condition in the first component $\mathcal{H}_{0}$ is the Titchmarsh-Weyl function of $T_{1}$ in the second component $\mathcal{H}_{1}$. This is well-known when performing interval truncation e.g. in numerics of Sturm-Liouville equations.

Remark 3.6 A natural generalization of the Robin type interface condition in (3.6) is

$\Gamma_{01} f_{0}=\Gamma_{11} f_{1}=\cdots=\Gamma_{n 1} f_{n}, \quad \Gamma_{01} f_{0}=\frac{1}{\tau_{0}} \Gamma_{02} f_{0}+\frac{1}{\tau_{1}} \Gamma_{12} f_{1}+\cdots+\frac{1}{\tau_{n}} \Gamma_{n 2} f_{n}$,

with coefficients $\tau_{j} \in \mathbb{R} \cup\{\infty\}, j=0,1, \ldots, n$. If all $\tau_{j}$ are positive or all $\tau_{j}$ are negative, the function $n_{0}$ which can be introduced in a similar way as above as

$$
n_{0}(z)=\frac{1}{\tau_{0}\left(1+\frac{1}{\tau_{1} m_{1}(z)}+\frac{1}{\tau_{2} m_{2}(z)}+\cdots+\frac{1}{\tau_{n} m_{n}(z)}\right)}, \quad z \in \mathbb{C} \backslash \mathbb{R},
$$

is a Nevanlinna function and induces a self-adjoint operator in some Hilbert space.

\section{The Reduced System and its Realization via de Branges' Inverse Theorem}

\subsection{The Reduced System}

In the following theorem we show that there exists a single linear relation $\widetilde{T}_{1}$ with Titchmarsh-Weyl function $\widetilde{m}_{1}=n_{0}$ such that the compressed resolvents on $\mathcal{H}_{0}$ of the corresponding self-adjoint extensions of $\mathbf{T}=T_{0} \oplus T_{1} \oplus \cdots \oplus T_{n}$ with interface condition (2.12) and of $\widetilde{\mathbf{T}}=T_{0} \oplus \widetilde{T}_{1}$ with continuity and Kirchhoff type conditions (3.9) coincide. The main tool here is an abstract inverse result [28].

Theorem 4.1 Let $T_{j}$ be symmetric linear relations in Hilbert spaces $\mathcal{H}_{j}$ with equal defect numbers $1, j=0,1, \ldots, n$, let the self-adjoint extension $\mathbf{T}_{\mathcal{A}, \mathcal{B}}$ of $\mathbf{T}=T_{0} \oplus$ $T_{1} \oplus \cdots \oplus T_{n}$ in $\mathcal{H}=\mathcal{H}_{0} \oplus \mathcal{H}_{1} \oplus \cdots \oplus \mathcal{H}_{n}$ be induced by (2.12) as in Theorem 3.1, and let $n_{0}$ be the Nevanlinna function in the boundary condition in (3.2). Then there exists a symmetric linear relation $\widetilde{T}_{1}$ in a Hilbert space $\widetilde{\mathcal{H}}_{1}$ with equal defect numbers 1, a 
boundary triplet $\left(\mathbb{C}, \widetilde{\Gamma}_{11}, \widetilde{\Gamma}_{12}\right)$ for $\widetilde{T}_{1}^{*}$ and Titchmarsh-Weyl function $\widetilde{m}_{1}=n_{0}$ such that for the self-adjoint extension $\widetilde{\mathbf{T}}_{\mathcal{A}_{0}, \mathcal{B}_{0}}$ of $\widetilde{\mathbf{T}}=T_{0} \oplus \widetilde{T}_{1}$ in $\widetilde{\mathcal{H}}=\mathcal{H}_{0} \oplus \widetilde{\mathcal{H}}_{1}$ with $\mathcal{A}_{0}$, $\mathcal{B}_{0}$ as in (3.10),

$$
\left.P_{0}\left(\mathbf{T}_{\mathcal{A}, \mathcal{B}}-z\right)^{-1}\right|_{\mathcal{H}_{0}}=\left.\widetilde{P}_{0}\left(\widetilde{\mathbf{T}}_{\mathcal{A}_{0}, \mathcal{B}_{0}}-z\right)^{-1}\right|_{\mathcal{H}_{0}}, \quad z \in \mathbb{C} \backslash \mathbb{R}
$$

here $P_{0}: \mathcal{H} \rightarrow \mathcal{H}_{0}, \widetilde{P}_{0}: \widetilde{\mathcal{H}} \rightarrow \mathcal{H}_{0}$ are the projections onto the first common component of $\mathcal{H}$ and $\widetilde{\mathcal{H}}$, respectively.

Proof According to a general inverse result for $Q$-functions (or Titchmarsh-Weyl functions), see [28], [11, Thm. 5.1], for the given Nevanlinna function $n_{0}$ in (3.3) there exists a symmetric linear relation $\widetilde{T}_{1}$ with equal defect numbers 1 and a boundary triplet $\left(\mathbb{C}, \widetilde{\Gamma}_{11}, \widetilde{\Gamma}_{12}\right)$ for $\widetilde{T}_{1}^{*}$ such that the corresponding Titchmarsh-Weyl function $\widetilde{m}_{1}$ coincides with $n_{0}$, i.e. $\tilde{m}_{1}=n_{0}$. On the other hand, by Remark 3.5 , the function $\tilde{n}_{0}$ in (3.2) for $\widetilde{\mathbf{T}}_{\mathcal{A}_{0}, \mathcal{B}_{0}}$ is given by $\widetilde{n}_{0}=\widetilde{m}_{1}$ and hence $\widetilde{n}_{0}=n_{0}$. Since $\mathbf{T}$ and $\widetilde{\mathbf{T}}$ have the same first component $T_{0}$, this implies that the problems (3.2) for $\mathbf{T}_{\mathcal{A}, \mathcal{B}}$ and $\widetilde{\mathbf{T}}_{\mathcal{A}_{0}, \mathcal{B}_{0}}$ coincide and hence Theorem 3.1 yields the claim.

Remark 4.2 For the special case that the interface conditions (2.12) in Theorem 4.1 are of Robin type (3.6), see Example 3.4, the relation $\tilde{m}_{1}=n_{0}$ takes the form

$$
\frac{1}{\widetilde{m}_{1}(z)}=\tau+\frac{1}{m_{1}(z)}+\frac{1}{m_{2}(z)}+\cdots+\frac{1}{m_{n}(z)}, \quad z \in \mathbb{C} \backslash \mathbb{R}
$$

Corollary 4.3 With the notation and assumptions of Theorem 4.1, we have:

(i) For $z \in \mathbb{C} \backslash \mathbb{R}$ and $f_{0} \in \mathcal{H}_{0}$, the first components of the solutions $\mathbf{g}$ and $\widetilde{\mathbf{g}}$ of the inhomogeneous equations

$$
\left(\mathbf{T}_{\mathcal{A}, \mathcal{B}}-z\right) \mathbf{g}=\left(\begin{array}{c}
f_{0} \\
0 \\
\vdots \\
0
\end{array}\right), \quad\left(\widetilde{\mathbf{T}}_{\mathcal{A}_{0}, \mathcal{B}_{0}}-z\right) \widetilde{\mathbf{g}}=\left(\begin{array}{c}
f_{0} \\
0
\end{array}\right)
$$

coincide, that is, $P_{0} \mathbf{g}=\widetilde{P}_{0} \widetilde{\mathbf{g}}$.

(ii) If $\lambda \in \sigma_{\mathrm{p}}\left(\mathbf{T}_{\mathcal{A}, \mathcal{B}}\right)$ with eigenvector $\mathbf{g}$ and $P_{0} \mathbf{g} \neq 0$, then $\lambda \in \sigma_{\mathrm{p}}\left(\widetilde{\mathbf{T}}_{\mathcal{A}_{0}, \mathcal{B}_{0}}\right)$ with eigenvector $\widetilde{\mathbf{g}}$ such that $\widetilde{P}_{0} \widetilde{\mathbf{g}}=P_{0} \mathbf{g}$, and vice versa if $\widetilde{P}_{0} \widetilde{\mathbf{g}} \neq 0$.

Proof The claim in (i) is immediate from Theorem 4.1. The claims in (ii) follow from the fact that for a self-adjoint operator $A$ in a Hilbert space with eigenvalue $\lambda_{0} \in \mathbb{R}$ the orthogonal projection $P_{\lambda_{0}}$ onto the corresponding eigenspace may be obtained as the strong limit $P_{\lambda_{0}}=\mathrm{s}-\lim _{z \rightarrow \lambda_{0}}\left(z_{0}-z\right)(A-z)^{-1}$ if $z$ tends to $\lambda_{0}$ perpendicularly to $\mathbb{R}$. 


\subsection{Implications for Cauchy Problems}

If $\mathbf{T}_{\mathcal{A}, \mathcal{B}}$ and $\widetilde{\mathbf{T}}_{\mathcal{A}_{0}, \mathcal{B}_{0}}$ are self-adjoint operators, they generate unitary groups $\left(\mathbf{U}_{\mathcal{A}, \mathcal{B}}(t)\right)_{t \in \mathbb{R}}$ and $\left(\widetilde{\mathbf{U}}_{\mathcal{A}_{0}, \mathcal{B}_{0}}(t)\right)_{t \in \mathbb{R}}$, respectively. The elements of these groups can be expressed as contour integrals with respect to the resolvents of the generators (see e.g. [21, IX.1]). Therefore the relation (4.1) in Theorem 4.1 readily implies

$$
\left.P_{0} \mathbf{U}_{\mathcal{A}, \mathcal{B}}(t)\right|_{\mathcal{H}_{0}}=\left.\widetilde{P}_{0} \widetilde{\mathbf{U}}_{\mathcal{A}_{0}, \mathcal{B}_{0}}(t)\right|_{\mathcal{H}_{0}}, \quad t \in \mathbb{R},
$$

that is, the compressions to $\mathcal{H}_{0}$ of the two groups coincide. A consequence of this is the following result on Cauchy problems for the operators $\mathbf{T}_{\mathcal{A}, \mathcal{B}}$ and $\widetilde{\mathbf{T}}_{\mathcal{A}_{0}, \mathcal{B}_{0}}$.

Theorem 4.4 Let $f_{0}: \mathbb{R} \rightarrow \mathcal{H}_{0}$ be continuously differentiable and let $x_{0} \in \mathcal{H}_{0}$ be such that $\mathbf{x}_{0}:=\left(\begin{array}{llll}x_{0} & 0 & \cdots & 0\end{array}\right)^{\mathrm{t}} \in \operatorname{dom} \mathbf{T}_{\mathcal{A}, \mathcal{B}}, \widetilde{\mathbf{x}}_{0}:=\left(\widetilde{x}_{0} 0\right)^{\mathrm{t}} \in \operatorname{dom} \widetilde{\mathbf{T}}_{\mathcal{A}_{0}, \mathcal{B}_{0}}$. Then the solutions of the Cauchy problem for $\mathbf{T}_{\mathcal{A}, \mathcal{B}}$ in $\mathcal{H}=\mathcal{H}_{0} \oplus \mathcal{H}_{1} \oplus \cdots \oplus \mathcal{H}_{n}$ given by

$$
\dot{\mathbf{x}}(t)-\mathrm{i} \mathbf{T}_{\mathcal{A}, \mathcal{B}} \mathbf{x}(t)=\left(\begin{array}{c}
f_{0}(t) \\
0 \\
\vdots \\
0
\end{array}\right)=: \mathbf{f}_{0}(t), \quad t \in \mathbb{R}, \quad \mathbf{x}(0)=\mathbf{x}_{0}=\left(\begin{array}{c}
x_{0} \\
0 \\
\vdots \\
0
\end{array}\right)
$$

and of the Cauchy problem for $\widetilde{\mathbf{T}}_{\mathcal{A}_{0}, \mathcal{B}_{0}}$ in $\widetilde{\mathcal{H}}=\mathcal{H}_{0} \oplus \widetilde{\mathcal{H}}_{1}$ with $\mathcal{A}_{0}$, $\mathcal{B}_{0}$ as in (3.10) given by

$$
\dot{\widetilde{\mathbf{x}}}(t)-\mathrm{i} \widetilde{\mathbf{T}}_{\mathcal{A}_{0}, \mathcal{B}_{0}} \widetilde{\mathbf{x}}(t)=\left(\begin{array}{c}
f_{0}(t) \\
0
\end{array}\right)=: \widetilde{\mathbf{f}}_{0}(t), \quad t \in \mathbb{R}, \quad \widetilde{\mathbf{x}}(0)=\widetilde{\mathbf{x}}_{0}=\left(\begin{array}{c}
x_{0} \\
0
\end{array}\right)
$$

have the same first components, i.e.

$$
P_{0} \mathbf{x}(t)=\widetilde{P}_{0} \widetilde{\mathbf{x}}(t), \quad t \in \mathbb{R} .
$$

Proof The solution $\mathbf{x}: \mathbb{R} \rightarrow \mathcal{H}$ of (4.3) is given by (see e.g. [21, Thm. IX.1.19], [24, Thm. I.6.1])

$$
\mathbf{x}(t)=\mathbf{U}_{\mathcal{A}, \mathcal{B}}(t) \mathbf{x}_{0}+\int_{0}^{t} \mathbf{U}_{\mathcal{A}, \mathcal{B}}(t-s) \mathbf{f}_{0}(s) \mathrm{d} s, \quad t \in \mathbb{R}
$$

a corresponding formula holds for the solution $\widetilde{\mathbf{x}}: \mathbb{R} \rightarrow \widetilde{\mathcal{H}}$ of (4.4). If we note that $P_{0} \mathbf{f}_{0}(t)=\widetilde{P}_{0} \widetilde{\mathbf{f}}_{0}(t)=f_{0}(t), t \in \mathbb{R}$, and $P_{0} \mathbf{x}_{0}=\widetilde{P}_{0} \widetilde{\mathbf{x}}_{0}=x_{0}$, claim (4.5) follows from (4.2).

Remark 4.5 (i) Clearly, the equality (4.5) continues to hold under more general assumptions on $x_{0}$ and $f_{0}$ if only the solutions can be expressed by a relation of the form (4.6), see e.g. [24, Thm. I.6.5].

ii) A corresponding result can be proved for the solutions of second order Cauchy problems if $\mathbf{T}_{\mathcal{A}, \mathcal{B}}$ and $\widetilde{\mathbf{T}}_{\mathcal{A}_{0}, \mathcal{B}_{0}}$ generate cosine families, see e.g. [14] and also [36, Props. 13.2.2 and 2.3.1]. 


\subsection{Realization by a Canonical System}

In this subsection we employ a fundamental inverse theorem by de Branges [7, Thm. 7], [38, Thm. 1] to show that the linear relation $\widetilde{T}_{1}$ in the reduced system in Theorem 4.1 can always be realized as a canonical system. To this end, we first need to recall some basic results for canonical systems on a single edge, where we omit the subscript $j$ for simplicity.

A Hermitian $H: e \rightarrow M_{2}(\mathbb{R})$ is a measurable real symmetric and non-negative $2 \times 2$-matrix function, defined on some interval (edge) $e=[0, \ell$ ) satisfying

$$
\operatorname{tr} H(x)=1, \quad x \in e \text { (a.e.). }
$$

Let $\mathcal{H}$ be the Hilbert space $\stackrel{\circ}{L}_{H}^{2}\left(e, \mathbb{C}^{2}\right)$ of measurable 2-vector functions on $e$ with inner product

$$
(f, g):=\int_{0}^{\ell}(H(x) f(x), g(x)) \mathrm{d} x, \quad f, g \in \stackrel{\circ}{L}_{H}^{2}\left(e, \mathbb{C}^{2}\right)
$$

arising from $L_{H}^{2}\left(e, \mathbb{C}^{2}\right)$ by forming the factor space with respect to $H$-indivisable intervals (see [17,38]). In $\stackrel{\circ}{L}_{H}^{2}\left(e, \mathbb{C}^{2}\right)$ we consider the symmetric linear relation $T$ defined as follows:

$$
\{f, \widehat{f}\} \in T, \quad f=\left(\begin{array}{ll}
f_{1} & f_{2}
\end{array}\right)^{\mathrm{t}}, \quad \widehat{f}=\left(\widehat{f_{1}} \widehat{f_{2}}\right)^{\mathrm{t}} \in \stackrel{\circ}{L}_{H}^{2}\left(e, \mathbb{C}^{2}\right),
$$

if and only if $f$ is absolutely continuous, $f$ satisfies the canonical differential equation with Hermitian $H$ given by

$$
-J f^{\prime}(x)=H(x) \widehat{f}(x), \quad x \in[0, \ell), \quad J:=\left(\begin{array}{cc}
0 & -1 \\
1 & 0
\end{array}\right),
$$

together with the boundary condition

$$
f(0)=\left(\begin{array}{l}
0 \\
0
\end{array}\right)
$$

and, if the edge is of finite length, i.e. $\ell<\infty$, a boundary condition

$$
\left(\begin{array}{ll}
\cos \alpha & \sin \alpha
\end{array}\right)\left(\begin{array}{l}
f_{1}(\ell) \\
f_{2}(\ell)
\end{array}\right)=0
$$

with some $\alpha \in[0, \pi)$; if $\ell=\infty$, no boundary condition at $\ell$ is needed. Note that also in the regular case $\ell<\infty$ the right endpoint $\ell$ can be replaced by $\infty$, see [38], if $H$ is extended to $[\ell, \infty)$ by

$$
H(x)=\left(\begin{array}{c}
\cos \alpha \\
\sin \alpha
\end{array}\right)(\cos \alpha \sin \alpha), \quad x \in[\ell, \infty) .
$$


Under these conditions $T$ is a symmetric relation in $\stackrel{\circ}{L}_{H}^{2}\left(e, \mathbb{C}^{2}\right)$ with equal defect numbers 1 and $T^{*}$ is the extension of $T$ obtained by omitting the boundary condition (4.8) at 0 .

We introduce the boundary functionals

$$
\Gamma_{1} f:=f_{1}(0), \quad \Gamma_{2} f:=f_{2}(0), \quad f=\left(f_{1} f_{2}\right)^{\mathrm{t}} \in \operatorname{dom} T^{*},
$$

and, for $z \in \mathbb{C}$, the fundamental matrix $Y(\cdot ; z)=\left(y_{i j}(\cdot ; z)\right)_{i, j=1}^{2}$, given as the $2 \times 2$ matrix function satisfying

$$
-J Y^{\prime}(x ; z)=z H(x) Y(x ; z), \quad x \in[0, \ell), \quad Y(0 ; z)=\mathcal{I}_{2} .
$$

Then, if $\ell<\infty$, the Titchmarsh-Weyl function $m$ is defined by the condition that the function

$$
h(\cdot ; z)=\left(u_{1}(\cdot ; z) u_{2}(\cdot ; z)\right)^{\mathrm{t}}:=Y(\cdot ; z)\left(\begin{array}{c}
-m(z) \\
1
\end{array}\right), \quad z \in \mathbb{C} \backslash \mathbb{R}
$$

satisfies the boundary condition (4.9) at $\ell$, which implies that

$$
m(z)=-\frac{y_{12}(\ell ; z) \cos \alpha+y_{22}(\ell ; z) \sin \alpha}{y_{11}(\ell ; z) \cos \alpha+y_{21}(\ell ; z) \sin \alpha}
$$

if $\ell=\infty$, then

$$
m(z)=-\lim _{x \rightarrow \infty} \frac{y_{12}(x ; z) \cos \alpha+y_{22}(x ; z) \sin \alpha}{y_{11}(x ; z) \cos \alpha+y_{21}(x ; z) \sin \alpha},
$$

where the limit exists and is independent of $\alpha \in[0, \pi)$. Note that for the canonical system (4.7) the relation (2.8) for $\zeta=z$ takes the form

$$
\frac{m(z)-\overline{m(z)}}{z-\bar{z}}=\int_{0}^{\ell}(H(x) h(x ; z), h(x ; z)) \mathrm{d} x, \quad z \in \mathbb{C} \backslash \mathbb{R},
$$

with $h(\cdot, z)$ given by $(4.11)$.

In the following theorem we make use of a fundamental inverse result of de Branges, see [7, Thm. 7], [38, Thm. 1]. Given any Nevanlinna function $m$, there exists a Hermitian $H$ on $[0, \infty)$ with the properties mentioned at the beginning of this subsection such that $m$ is the Titchmarsh-Weyl function of the canonical system corresponding to $H$. This means that the symmetric linear relation in Theorem 4.1 can always be chosen to be a canonical system.

Theorem 4.6 The linear relation $\widetilde{\mathbf{T}}_{\mathcal{A}_{0}, \mathcal{B}_{0}}$ in Theorem 4.1 can be chosen as follows. There exists a Hermitian $\widetilde{H}_{1}$ on $\widetilde{e}_{1}=[0, \infty)$ such that the corresponding canonical system which defines $\widetilde{T}_{1}$ in $\widetilde{\mathcal{H}}_{1}=\stackrel{\circ}{L}_{\widetilde{H}_{1}}^{2}\left(\widetilde{e}_{1}, \mathbb{C}^{2}\right)$ has Titchmarsh-Weyl function $\widetilde{m}_{1}=n_{0}$ 
and $\widetilde{\mathbf{T}}_{\mathcal{A}_{0}, \mathcal{B}_{0}}$ is the restriction of $\widetilde{\mathbf{T}}^{*}=T_{0}^{*} \oplus \widetilde{T}_{1}^{*}$ in $\widetilde{\mathcal{H}}=\mathcal{H}_{0} \oplus L_{\widetilde{H}_{1}}^{2}\left(\widetilde{e}_{1}, \mathbb{C}^{2}\right)$ by the interface condition

$$
\left(\begin{array}{cc}
1 & -1 \\
0 & 0
\end{array}\right)\left(\begin{array}{c}
\Gamma_{01} f_{0} \\
\widetilde{f}_{11}(0)
\end{array}\right)+\left(\begin{array}{ll}
0 & 0 \\
1 & 1
\end{array}\right)\left(\begin{array}{c}
\Gamma_{02} f_{0} \\
\widetilde{f}_{12}(0)
\end{array}\right)=0
$$

$\operatorname{for}\left(\begin{array}{lll}f_{0} & \widetilde{f}_{1}\end{array}\right)^{\mathrm{t}} \in \operatorname{dom} \widetilde{\mathbf{T}}^{*}, \widetilde{f_{1}}=\left(\begin{array}{ll}\tilde{f}_{11} & \widetilde{f}_{12}\end{array}\right)^{\mathrm{t}}$.

Proof The claims follow from Theorem 4.1 and from de Branges' theorem (see [7, Thm. 7], [38, Thm. 1]).

\section{Differential Operators on Star Graphs}

In this section we investigate the structure of the reduced problem if the symmetric relations $T_{j}, j=0,1, \ldots, n$, (or some of them) are differential or difference operators on the edges of a star graph $\mathcal{G}$. In particular, we ask e.g. the following question: If all differential operators are of one type, when can the reduced system constructed in the previous section be chosen to consist of two differential operators of the same type?

We denote the central vertex of $\mathcal{G}$ by $v$ and the outer vertices by $v_{0}, v_{1}, \ldots, v_{n}$ (see Fig. 2 below); the coordinate $x_{j}$ on the edge $e_{j}$ increases from 0 at $v$ to $\ell_{j}$ at the outer vertex $v_{j}, j=0,1, \ldots, n$.

The symmetric relations $T_{j}, j=0,1, \ldots, n$, in the Hilbert spaces $\mathcal{H}_{j}$ may be induced by three different types of differential or difference expressions on the edges $e_{j}$, which are all supposed to be regular at $v$ : canonical systems, Sturm-Liouville problems, and Krein strings; they include, as special cases, Dirac systems and Stieltjes strings. Here the 'interface' condition (2.12) becomes a matching condition at the common vertex $v$.

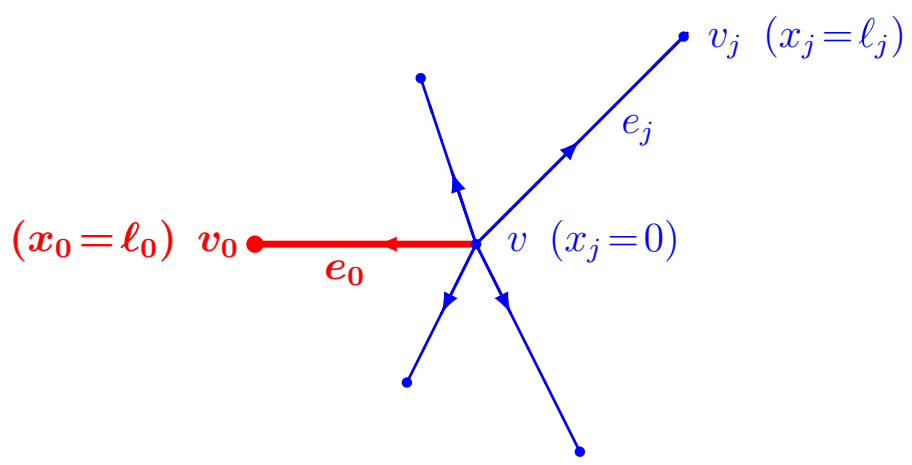

Fig. 2 Star graph $\mathcal{G}$ with $n+1$ edges and distinguished edge $e_{0}$ (color figure online) 


\subsection{Canonical Systems and Sturm-Liouville Operators}

By Theorem 4.6, independently of the type of differential operators inducing the relations $T_{j}, j=1,2, \ldots, n$ on the subgraph of edges different from $e_{0}$ (the blue/nonbold part in Fig. 2), the new part $\widetilde{T}_{1}$ of the reduced system $\widetilde{\mathbf{T}}_{\mathcal{A}_{0}, \mathcal{B}_{0}}$ extending $T_{0} \oplus \widetilde{T}_{1}$ can always be chosen to be a canonical system on an edge $\widetilde{e}_{1}$.

Thus the answer to the question when the whole reduced system consists of two canonical systems is immediate from Theorem 4.6: it suffices that only $T_{0}$ is induced by a canonical system.

Corollary 5.1 Suppose that in Theorem 4.6 the symmetric relation $T_{0}$ is given by a canonical system on an edge $e_{0}$ with Hermitian $H_{0}$ and that the other symmetric relations $T_{j}, j=1,2, \ldots, n$, therein are arbitrary. Then the reduced system $\widetilde{\mathbf{T}}_{\mathcal{A}_{0}, \mathcal{B}_{0}}$ can be chosen to consist of two canonical systems on the path graph $\widetilde{\mathcal{G}}$ with the two edges $e_{0}, \widetilde{e}_{1}$ with Hermitians $H_{0}, \widetilde{H}_{1}$, respectively, and by the interface condition (4.14) which takes the form

$$
f_{01}(0)=\widetilde{f}_{11}(0), \quad f_{02}(0)=-\widetilde{f}_{12}(0)
$$

for $f=\left(f_{0} \tilde{f}_{1}\right)^{\mathrm{t}} \in \operatorname{dom} \widetilde{\mathbf{T}}^{*}, f_{0}=\left(f_{01} f_{02}\right)^{\mathrm{t}}, \widetilde{f}_{1}=\left(\tilde{f}_{11} \tilde{f}_{12}\right)^{\mathrm{t}}$ (see Fig. 3 below).

We mention that two canonical systems coupled at a common end-point, as obtained in Corollary 5.1, were studied in [17, Sect. 6].

For Sturm-Liouville operators the situation is very different. Since there are no suitable inverse results in this case, one cannot hope to be able to reduce a system of Sturm-Liouville operators on a star graph with $n+1$ edges to a system of two SturmLiouville operators on a path graph. Nevertheless the results of the two previous sections can still be applied.

To explain this, suppose that the symmetric relations $T_{j}, j=0,1, \ldots, n$, are given by Sturm-Liouville operators in the Hilbert spaces $\mathcal{H}_{j}=L^{2}\left(e_{j}, \mathbb{C}\right)$,

$$
\left(T_{j} y_{j}\right)\left(x_{j}\right):=-\frac{\mathrm{d}^{2} y_{j}\left(x_{j}\right)}{\mathrm{d} x^{2}}+q_{j}\left(x_{j}\right) y_{j}\left(x_{j}\right), \quad x_{j} \in e_{j}=\left[0, \ell_{j}\right],
$$

where $q_{j}$ is real-valued and locally summable on $\left[0, \ell_{j}\right)$, with boundary conditions

$$
y_{j}(0)=y_{j}^{\prime}(0)=0
$$

at $v$ and, if $T_{j}$ is regular at $v_{j}$,

$$
\begin{gathered}
\cos \alpha_{j} y_{j}\left(\ell_{j}\right)+\sin \alpha_{j} y^{\prime}\left(\ell_{j}\right)=0 \\
\left(x_{0}=\ell_{0}\right) \quad v_{0} \underset{e_{0}}{v\left(x_{0}=\widetilde{x}_{1}=0\right)} \underset{\widetilde{e}_{1}}{v} \cdot \widetilde{v}_{1}\left(\widetilde{x}_{1}=\infty\right)
\end{gathered}
$$

Fig. 3 Reduced path graph of two canonical systems (color figure online) 
with some $\alpha_{j} \in[0, \pi)$. If $T_{j}$ is singular at $v_{j}$, the boundary condition at $v_{j}$ is either omitted (in the limit point case), or has to be modified to a limit circle type boundary condition. In any case, under these conditions $T_{j}$ is a symmetric operator in $L^{2}\left(e_{j}, \mathbb{C}\right)$ with equal defect numbers 1 and the operator $T_{j}^{*}$ is the extension of $T_{j}$ obtained by omitting the boundary condition (5.1) at $v$.

We introduce the boundary functionals

$$
\Gamma_{j 1} y_{j}:=y_{j}(0), \quad \Gamma_{j 2} y_{J}:=y_{j}^{\prime}(0), \quad y \in \operatorname{dom} T_{j}^{*},
$$

and, for $z \in \mathbb{C}$, the fundamental system $\varphi_{j}(\cdot ; z), \psi_{j}(\cdot ; z)$ of the homogeneous equation

$$
-\frac{\mathrm{d}^{2} y_{j}(x)}{\mathrm{d} x^{2}}+q_{j}(x) y_{j}(x)-z y_{j}(x)=0, \quad x \in e_{j},
$$

satisfying

$$
\varphi_{j}(0 ; z)=1, \quad \varphi_{j}^{\prime}(0 ; z)=0 ; \quad \psi_{j}(0 ; z)=0, \quad \psi_{j}^{\prime}(0 ; z)=1
$$

Then the Titchmarsh-Weyl coefficient $m_{j}$ is determined by the condition that the solution $\chi_{j}(\cdot ; z)=-\psi_{j}(\cdot ; z)+m_{j}(z) \varphi_{j}(\cdot ; z)$ satisfies the boundary condition (5.2) at $v_{j}$, that is,

$$
m_{j}(z)=\frac{\cos \alpha_{j} \psi_{j}\left(\ell_{j} ; z\right)+\sin \alpha_{j} \psi_{j}^{\prime}\left(\ell_{j} ; z\right)}{\cos \alpha_{j} \varphi_{j}\left(\ell_{j} ; z\right)+\sin \alpha_{j} \varphi_{j}^{\prime}\left(\ell_{j} ; z\right)}, \quad z \in \mathbb{C} \backslash \mathbb{R}
$$

We mention that here the relation (2.8) takes the form

$$
\frac{m_{j}(z)-\overline{m_{j}(z)}}{z-\bar{z}}=\int_{0}^{\ell_{j}}\left|\chi_{j}(x ; z)\right|^{2} \mathrm{~d} x, \quad z \in \mathbb{C} \backslash \mathbb{R}, \quad j=0,1, \ldots, n .
$$

In this situation Theorem 3.1 as well as Theorem 4.6 apply and yield the following reductions:

Theorem 3.1 reduces the system of Sturm-Liouville operators $T_{j}, j=0,1, \ldots, n$, on the star graph $\mathcal{G}$ with arbitrary interface conditions (2.12) to the Sturm-Liouville operator $T_{0}$ on the single edge $e_{0}$ with an energy depending boundary condition at $v$ given by (3.3) with $m_{j}$ as in (5.6), $j=1,2, \ldots, n$.

According to Theorem 4.6, the energy depending boundary condition at $v$ can be replaced by attaching a canonical system on a new edge $\widetilde{e}_{1}$. In order to decide whether the latter can be replaced by a Sturm-Liouville operator would require suitable inverse results for Sturm-Liouville problems, which to the best of our knowledge do not exist.

\subsection{Krein Strings and Stieltjes Strings}

For a star graph of $n+1$ Krein strings, the reduced system need not consist of two Krein strings. For the case of Robin type interface conditions, we establish a sufficient 
condition involving the Titchmarsh-Weyl functions of the $n$ Krein strings of the subsystem and the Robin parameter. In this subsection we strongly rely on [18] (see also [20]).

A Krein string is given by a bounded left-continuous and non-decreasing function $M$ on an (edge) interval $e=[0, \ell), 0<\ell \leq \infty$; here, for $x \in(0, \ell)$, the value $M(x)$ is considered to be the mass of the string on the interval $[0, x)$. We assume that the string has no concentrated mass at 0 and at $\ell$ so that, in particular, $M(0)=M(0+)=0$, and we set $M(\ell):=\lim _{x \nearrow \ell} M(x) \leq \infty$.

By $\mathcal{H}=L_{M}^{2}(e, \mathbb{C})$ we denote the Hilbert space of measurable functions $f$ on $e$ with inner product

$$
(f, g):=\int_{0}^{\ell} f(x) \overline{g(x)} \mathrm{d} M(x), \quad f, g \in L_{M}^{2}(e, \mathbb{C}) .
$$

In order to realize the symmetric relation $T$ in $L_{M}^{2}(e, \mathbb{C})$, we have to distinguish the two cases that $\ell$ is a regular boundary point, i.e. $\ell+M(\ell)<\infty$, or that $\ell$ is a singular boundary point, i.e. $\ell+M(\ell)=\infty$.

(i) $\underline{\ell+M(\ell)<\infty}$ : For $f, g \in L_{M}^{2}(e, \mathbb{C})$, we define

$$
f \in \operatorname{dom} T, \quad T f=g: \Longleftrightarrow\left\{\begin{array}{l}
f(x)=-\int_{0}^{x}(x-t) g(t) \mathrm{d} M(t), \quad x \in e, \\
\cos \alpha f(\ell)+\sin \alpha f^{\prime}(\ell)=0,
\end{array}\right.
$$

with some $\alpha \in[0, \pi)$. Due to (5.7) every $f \in \operatorname{dom} T$ is absolutely continuous with left and right derivatives

$$
f_{ \pm}^{\prime}(x)=-\int_{0}^{x \pm 0} g(t) \mathrm{d} M(t), \quad x \in(0, \ell)
$$

and $f(0)=f^{\prime}(0)=0$. Note that, formally, the first equation on the right hand side of (5.7) reads

$$
-\frac{\mathrm{d}}{\mathrm{d} M(x)} \frac{\mathrm{d} f(x)}{\mathrm{d} x}=g(x), \quad x \in e .
$$

It is not difficult to check that $T$ is symmetric but not necessarily densely defined and that, with $f, g \in L_{M}^{2}(e, \mathbb{C})$, the adjoint operator or relation $T^{*}$ is given by

$$
f \in \operatorname{dom} T^{*}, \quad T^{*} f=g \Longleftrightarrow\left\{\begin{array}{l}
f(x)=c+d x-\int_{0}^{x}(x-t) g(t) \mathrm{d} M(t), \quad x \in e, \\
\cos \alpha f(\ell)+\sin \alpha f^{\prime}(\ell)=0,
\end{array}\right.
$$

with $c, d \in \mathbb{C}$. Hence $T$ has equal defect numbers 1 . For $f \in \operatorname{dom} T^{*}$ as in (5.8) we introduce the boundary functionals

$$
\Gamma_{1} f:=c=f(0), \quad \Gamma_{2} f:=d=f^{\prime}(0) .
$$


A fundamental role is played by the solutions $\varphi(\cdot ; z), \psi(\cdot ; z)$ of the integral equations

$$
\begin{aligned}
& \varphi(x ; z)=1-z \int_{0}^{x}(x-t) \varphi(t ; z) \mathrm{d} M(t), \quad x \in e, \\
& \psi(x ; z)=x-z \int_{0}^{x}(x-t) \psi(t ; z) \mathrm{d} M(t), \quad x \in e .
\end{aligned}
$$

For every $z \in \mathbb{C}$, they form a fundamental system of the (formal) homogeneous equation

$$
-\frac{\mathrm{d}}{\mathrm{d} M(x)} \frac{\mathrm{d} f(x)}{\mathrm{d} x}-z f(x)=0, \quad x \in e .
$$

Then, in the regular case, the Titchmarsh-Weyl function $m$ corresponding to the boundary triplet with $\Gamma_{1}, \Gamma_{2}$ given by (5.9), i.e. for the boundary condition $\Gamma_{2} f=f^{\prime}(0)=0$ is defined by the property that the function $-\psi(\cdot ; z)+m(z) \varphi(\cdot ; z)$ satisfies the boundary condition at $\ell$, and hence

$$
m(z)=\frac{\cos \alpha \psi(\ell ; z)+\sin \alpha \psi^{\prime}(\ell ; z)}{\cos \alpha \varphi(\ell ; z)+\sin \alpha \varphi^{\prime}(\ell ; z)}, \quad z \in \mathbb{C} \backslash \mathbb{R}
$$

Recall that a function $F$ belongs to the Stieltjes class $\mathcal{S}$ or is a Stieltjes function if $F$ is a Nevanlinna function that is holomorphic also on $(-\infty, 0)$ with $F(x) \geq 0$ for $x \in(-\infty, 0)$, see [19]. According to [19, Thm. 5.2], $F \in \mathcal{S}$ if and only if $F$ has an integral representation

$$
F(z)=\gamma+\int_{0-}^{\infty} \frac{\mathrm{d} \sigma(\lambda)}{\lambda-z}, \quad z \in \mathbb{C} \backslash[0, \infty),
$$

with some $\gamma \geq 0$ and a measure $\sigma$ on $[0, \infty)$ with $\int_{0-}^{\infty} \frac{\mathrm{d} \sigma(x)}{1+x}<\infty$. Since $F \in \mathcal{S}$ is non-decreasing on $(-\infty, 0)$, the limits at the boundary points exist,

$$
\lim _{x \rightarrow-\infty} F(x)=\gamma, \quad F(0-)=\lim _{x \nearrow 0} F(x) \in[0, \infty]
$$

It is not difficult to see that, with the representation (5.11),

$$
\lim _{x \rightarrow-\infty}(-x) \int_{0-}^{\infty} \frac{\mathrm{d} \sigma(\lambda)}{\lambda-x}=\int_{0-}^{\infty} \mathrm{d} \sigma(\lambda)(\leq \infty) .
$$

For the function $m$ in (5.10), from [18, Thm. 9.1 and its proof] it follows that

$$
m \in \mathcal{S} \Longleftrightarrow \alpha \in[0, \pi / 2]
$$

which, in turn, is equivalent to the property that the self-adjoint relation $T_{0,1}$ with the boundary conditions in (5.7) at $\ell$ and $f^{\prime}(0)=0$ at $v$ is non-negative. Note that in 
our case the function $N$ in the representation [18, (9.1)] is constant, $N(z) \equiv \tan \alpha \in$ $[0,+\infty]$, and hence belongs to the extended Stieltjes class $\widetilde{\mathcal{S}}=\mathcal{S} \cup\{\infty\}$.

(ii) $\ell+M(\ell)=\infty$ : In the singular case we have to distinguish two subcases. If $\int_{0}^{\ell} x^{2} \mathrm{~d} M(x)=\infty$, then the limit point case prevails at $\ell$ and no boundary condition at $\ell$ is needed. The Krein string with the boundary condition $f^{\prime}(0)=0$ induces a unique self-adjoint operator $S$ in $L_{M}^{2}(e, \mathbb{C})$ which is non-negative. The corresponding Titchmarsh-Weyl function belongs to the Stieltjes class $\mathcal{S}$, see [18, Thm. 10.1]. If $\int_{0}^{\ell} x^{2} \mathrm{~d} M(x)<\infty$, then the limit circle case prevails at $\ell$. In this case, the Krein string with the boundary condition $f^{\prime}(0)=0$ induces infinitely many self-adjoint operators in $L_{M}^{2}(e, \mathbb{C})$, but only one non-negative self-adjoint operator $S$ in $L_{M}^{2}(e, \mathbb{C})$.

In both cases, we define the symmetric operator $T$ in $L_{M}^{2}(e, \mathbb{C})$ as

$$
T:=\left.S\right|_{\operatorname{dom}(T)}, \quad \operatorname{dom}(T):=\{f \in \operatorname{dom}(S): f(0)=0\}
$$

then $T$ has equal defect numbers 1 and $T$ is non-negative because so is $S$. With the boundary functionals $\Gamma_{1}, \Gamma_{2}$ as in (5.9), the self-adjoint extension $T_{0,1}=S$ of $T$ is given by the boundary condition $\Gamma_{2} f=f^{\prime}(0)=0$ and the corresponding TitchmarshWeyl function $m$ is a Stieltjes function.

By an inverse result of Krein, see [18, Thm. 11.2] and [12, Sect. 5.8], every Stieltjes function is the Titchmarsh-Weyl function of a Krein string on an interval $[0, \ell$ ) with the boundary condition

$$
f_{-}^{\prime}(0)=0
$$

at the left endpoint 0 , and either with singular right endpoint $\ell$, i.e. $\ell+M(\ell)=\infty$, or with regular endpoint $\ell$ and with a boundary condition

$$
\cos \alpha f(\ell)+\sin \alpha f^{\prime}(\ell)=0
$$

with some $\alpha \in[0, \pi / 2]$. In this correspondence, $\gamma>0$ in the representation (5.11) for $m$ in (5.10) means that the interval $[0, \gamma)$ is free of mass, i.e. $M(0)=M(\gamma)=0$.

As a consequence of this inverse result, if the function $n_{0}$ from (3.3) in Theorem 3.1 belongs to the class $\mathcal{S}$, then the operator $\widetilde{T}_{1}$ can be chosen to be a Krein string on some interval $\widetilde{e}_{1}=\left[0, \widetilde{\ell}_{1}\right)$. In the following theorem we establish a sufficient condition for $n_{0} \in \mathcal{S}$ for the case of Robin type interface conditions.

Theorem 5.2 Suppose that in Theorem 4.1 the linear relations $T_{j}, j=1,2, \ldots, n$, are given by Krein strings with mass distributions $M_{j}$ on the edges $e_{j}$ of the star graph $\mathcal{G}$ so that $M_{j}(0+)=0$. If the vertex $v_{j}$ is regular, $T_{j}$ is defined as in (5.7) with $\alpha_{j} \in[0, \pi / 2]$ in the boundary condition at $v_{j}$; if the vertex $v_{j}$ is singular, $T_{j}$ is defined as in (5.14). In both cases, we denote by $m_{j}, j=1,2, \ldots, n$, the TitchmarshWeyl functions corresponding to the boundary condition $f_{j}^{\prime}(0)=0$. Further, let the self-adjoint extension $\mathbf{T}_{\mathcal{A}_{\tau}, \mathcal{B}_{\tau}}$ of $\mathbf{T}=T_{0} \oplus T_{1} \oplus \cdots \oplus T_{n}$ be given by a Robin type interface condition (3.6) at $v$ with $\tau \in \mathbb{R}$. If

$$
-\frac{1}{m_{1}(0-)}-\frac{1}{m_{2}(0-)}-\cdots-\frac{1}{m_{n}(0-)} \leq \tau,
$$


then the linear relation $\widetilde{T}_{1}$ in Theorem 4.1 can be chosen to be a Krein string on some edge $\widetilde{e}_{1}=\left[0, \widetilde{\ell}_{1}\right)$ with boundary triplet $\left(\mathbb{C}, \widetilde{\Gamma}_{11}, \widetilde{\Gamma}_{12}\right)$ as in $(5.9)$, and the right endpoint being either singular or subject to a boundary condition (5.16) for some $\widetilde{\alpha} \in[0, \pi / 2]$. The Krein string $\widetilde{T}_{1}$ has no concentrated mass at 0 , and it has a massfree interval at 0 if and only if all the Krein strings $T_{j}, j=1,2, \ldots, n$, have mass-free intervals at 0 . The operator $\widetilde{\mathbf{T}}$ in Theorem 4.1 is the restriction of $\widetilde{\mathbf{T}}^{*}=T_{0}^{*} \oplus \widetilde{T}_{1}^{*}$ by the interface condition

$$
\Gamma_{01} f_{0}=\widetilde{f}_{1}(0), \quad \Gamma_{02} f_{0}=-\widetilde{f}_{1}^{\prime}(0) .
$$

If the relation $T_{0}$ is also given by a Krein string, then $\widetilde{\mathbf{T}}$ consists of two Krein strings on the graph $\widetilde{\mathcal{G}}$ with the interface conditions $f_{0}(0)=\widetilde{f}_{1}(0),\left(f_{0}\right)^{\prime}(0)=-\left(\widetilde{f}_{1}\right)^{\prime}(0)$.

Proof According to (3.8), the Nevanlinna function $n_{0}$ in (3.3) for the case of Robin type interface conditions (3.6) has the form

$$
n_{0}(z)=\frac{1}{\tau+\frac{1}{m_{1}(z)}+\frac{1}{m_{2}(z)}+\cdots+\frac{1}{m_{n}(z)}}, \quad z \in \mathbb{C} \backslash \mathbb{R}
$$

For $j=1,2, \ldots, n$, the functions $m_{j}$ belong to $\mathcal{S}$; in the regular case this is guaranteed by the condition $\alpha_{j} \in[0, \pi / 2]$ in the boundary condition in (5.7), in the singular case this is ensured by the choice of $T_{j ; 0,1}$. Thus $n_{0}$ is holomorphic on $(-\infty, 0)$ since so are $m_{1}, m_{2}, \ldots, m_{n}$, and $n_{0}(x)>0$ for $x \in(-\infty, 0)$ if and only if

$$
\tau+\frac{1}{m_{1}(x)}+\frac{1}{m_{2}(x)}+\cdots+\frac{1}{m_{n}(x)}>0, \quad x \in(-\infty, 0) .
$$

Because $m_{1}, m_{2}, \ldots, m_{n}$ are increasing on $(-\infty, 0),(5.19)$ is equivalent to (5.17). Therefore (5.17) implies $n_{0} \in \mathcal{S}$, and according to the inverse result of Krein mentioned above, $\widetilde{T}_{1}$ with Titchmarsh-Weyl function $\widetilde{m}_{1}=n_{0}$ can be chosen a (regular or singular) Krein string.

Next we prove the claim about the mass-free interval of $\widetilde{T}_{1}$. Since $\widetilde{m}_{1}=n_{0}$ and $m_{j}, j=1,2, \ldots, n$, belong to $\mathcal{S}$, by (5.11) they have representations

$$
\tilde{m}_{1}(z)=\tilde{\gamma}_{1}+\int_{0-}^{\infty} \frac{\mathrm{d} \widetilde{\sigma}_{1}(\lambda)}{\lambda-z}, \quad m_{j}(z)=\gamma_{j}+\int_{0-}^{\infty} \frac{\mathrm{d} \sigma_{j}(\lambda)}{\lambda-z}, \quad z \in \mathbb{C} \backslash[0, \infty),
$$

with $\tilde{\gamma}_{1}, \gamma_{j} \geq 0, j=1,2, \ldots, n$. Then the first relation in (5.12) and (5.18) show that

$$
\tilde{\gamma}_{1}=\frac{1}{\tau+\frac{1}{\gamma_{1}}+\frac{1}{\gamma_{2}}+\cdots+\frac{1}{\gamma_{n}}}
$$

Because $\tau \neq \infty$, this implies that $\widetilde{\gamma}_{1}>0$ if and only if all $\gamma_{j}>0, j=1,2, \ldots, n$, which proves the claim about the mass-free interval of $\widetilde{T}_{1}$. 
To prove that the concentrated mass $\widetilde{M}_{1}(0+)$ of $\widetilde{T}_{1}$ at 0 equals 0 , we first note that (see $\left.\left[18,11.2^{\circ}\right]\right)$

$$
\frac{1}{\widetilde{M}_{1}(0+)}=\int_{0-}^{\infty} \mathrm{d} \widetilde{\sigma}_{1}(\lambda) .
$$

Set $J_{0}:=\left\{j \in\{1,2, \ldots, n\}: \gamma_{j}=0\right\}$. If $J_{0}=\emptyset$, then $\tilde{\gamma}_{1}>0$ and hence there is no concentrated mass 0 . If $J_{0} \neq \emptyset$, then $\tilde{\gamma}_{1}=0$. Then the relations (5.21), (5.13) and the first relation in (5.20) imply that

$$
\begin{aligned}
\frac{1}{\widetilde{M}_{1}(0+)} & =\lim _{x \rightarrow-\infty}(-x) \tilde{m}_{1}(x) \\
& =\lim _{x \rightarrow-\infty} \frac{-x}{\tau+\frac{1}{m_{1}(x)}+\frac{1}{m_{2}(x)}+\cdots+\frac{1}{m_{n}(x)}} \\
& =\lim _{x \rightarrow-\infty} \frac{1}{\frac{\tau}{-x}+\sum_{j \in J_{0}} \frac{1}{(-x) m_{j}(x)}+\sum_{j \notin J_{0}} \frac{1}{(-x) m_{j}(x)}} .
\end{aligned}
$$

Clearly, the first term in the denominator tends to 0 ; for the other terms we have

$$
\begin{array}{ll}
(-x) m_{j}(x) \rightarrow \int_{0-}^{\infty} \mathrm{d} \sigma_{j}(\lambda)=\frac{1}{M_{j}(0+)}=\infty, & x \rightarrow-\infty, \quad \text { for } j \in J_{0}, \\
(-x) m_{j}(x)=-x \gamma_{j}-x \int_{0-}^{\infty} \frac{\mathrm{d} \sigma(\lambda)}{\lambda-x} \rightarrow \infty, & x \rightarrow-\infty, \quad \text { for } j \notin J_{0} .
\end{array}
$$

Altogether, this proves $\tilde{M}_{1}(0+)=0$.

Finally, if $T_{0}$ is also supposed to be given by a Krein string, then the last claim follows from the fact that Kirchhoff conditions on the vertex $v$ joining $e_{0}$ and $\widetilde{e}_{1}$ amount to the required continuity conditions at $v$.

Remark 5.3 A Krein string with mass distribution function $M$ describes a gap diffusion with state space supp $M$ and speed measure $M$ (comp. [27]). Hence, for a given system of gap diffusions on the graph $\mathcal{G}$ with state space $\mathcal{X}:=\bigcup_{j=0}^{n}$ supp $M_{j}$, Theorem 5.2 provides a system of two gap diffusions on the path graph $\widetilde{\mathcal{G}}$ with state space $\widetilde{\mathcal{X}}:=\operatorname{supp} M_{0} \cup \operatorname{supp} \widetilde{M}_{1}$. According to relation (4.2), the corresponding transition probabilities $P(t ; x, \Delta), t>0, x \in \mathcal{X}, \Delta \subset \mathcal{X}, \Delta$ measurable, and $\widetilde{P}(t ; \tilde{x}, \widetilde{\Delta}), t>0$, $\widetilde{x} \in \widetilde{\mathcal{X}}, \widetilde{\Delta} \subset \widetilde{\mathcal{X}}, \widetilde{\Delta}$ measurable, satisfy

$P(t ; x, \Delta)=\widetilde{P}(t ; x, \Delta), \quad t>0, \quad x \in \operatorname{supp} M_{0}, \quad \Delta \subset \operatorname{supp} M_{0}, \quad \Delta$ measurable.

A Krein string is called a Stieltjes string (see [18, Sect. 13]) if the mass distribution function $M$ is a step function with jumps $\mu_{k}>0$ at points $l_{0}+l_{1}+\cdots+l_{k-1}$, $k=1,2, \ldots$, where $l_{0} \geq 0$ is the length of the possibly mass-free interval at 0 and 
$l_{k}>0$ is the length of the interval between the masses $\mu_{k}$ and $\mu_{k+1}, k=1,2, \ldots$. Then the Titchmarsh-Weyl function admits a continued fraction expansion

$$
m(z)=l_{0}-\frac{1}{\mu_{1} z+\frac{1}{l_{1}-\frac{1}{\mu_{2} z+\ddots}}}, \quad z \in \mathbb{C} \backslash \mathbb{R} .
$$

On the other hand, such a continued fraction representation holds if and only if, for every $k \in \mathbb{N}$, the Titchmarsh-Weyl function $m$ admits an expansion

$$
m(z)=l_{0}-\frac{s_{0}}{z}-\frac{s_{1}}{z^{2}}-\cdots-\frac{s_{k}}{z^{k+1}}+\mathrm{o}\left(\frac{1}{z^{k+1}}\right), \quad z \rightarrow-\infty,
$$

with coefficients $s_{k} \geq 0$ which are the moments of $\sigma, s_{k}=\int_{0-}^{\infty} \lambda^{k} \mathrm{~d} \sigma(\lambda), k=0,1, \ldots$ (see [18, Sect. 13], [15]). It is not difficult to see that if the Titchmarsh-Weyl functions $m_{j}, j=1,2, \ldots, n$, in Theorem 5.2 admit expansions (5.22), then so does the function $n_{0}$ in (5.18) and hence the following result is immediate from Theorem 5.2.

Corollary 5.4 If in Theorem 5.2 the Krein strings on $e_{j}$ inducing $T_{j}, j=1,2, \ldots, n$, are Stieltjes strings, then also the Krein string on $\widetilde{e}_{1}$ inducing $\widetilde{T}_{1}$ with Titchmarsh-Weyl function $\widetilde{m}_{1}=n_{0}$ is a Stieltjes string. Its masses $\widetilde{\mu}_{k}, k=1,2, \ldots$, and its lengths $\widetilde{l}_{k}$, $k=1,2, \ldots$, can be obtained from the continued fraction expansion

$$
n_{0}(z)=\tilde{l}_{0}-\frac{1}{\tilde{\mu}_{1} z+\frac{1}{\tilde{l}_{1}-\frac{1}{\tilde{\mu}_{2} z+\ddots}}}, \quad z \in \mathbb{C} \backslash \mathbb{R}
$$

of the Titchmarsh-Weyl function $n_{0}$ given by (5.18).

Acknowledgements Open access funding provided by TU Wien (TUW). All authors thank for the support of EPSRC, Grant No. EP/1038217/1, which enabled this work to commence in January 2013. The last author gratefully acknowledges the support of the Swiss National Science Foundation, SNF, Grant No. 169104.

Open Access This article is distributed under the terms of the Creative Commons Attribution 4.0 International License (http://creativecommons.org/licenses/by/4.0/), which permits unrestricted use, distribution, and reproduction in any medium, provided you give appropriate credit to the original author(s) and the source, provide a link to the Creative Commons license, and indicate if changes were made.

\section{References}

1. Adamyan, V., Langer, H., Tretter, C., Winklmeier, M.: Dirac-Krein systems on star graphs. Integral Equ. Oper. Theory 86(1), 121-150 (2016)

2. Avdonin, S., Kurasov, P.: Inverse problems for quantum trees. Inverse Probl. Imaging 2(1), 1-21 (2008)

3. Avdonin, S., Kurasov, P., Nowaczyk, M.: Inverse problems for quantum trees II: recovering matching conditions for star graphs. Inverse Probl. Imaging 4(4), 579-598 (2010) 
4. Avdonin, S., Nicaise, S.: Source identification problems for the wave equation on graphs. Inverse Probl. 31(9), 095007, 29 (2015)

5. Berkolaiko, G., Kuchment, P.: Introduction to Quantum Graphs, Mathematical Surveys and Monographs, vol. 186. American Mathematical Society, Providence (2013)

6. Calkin, J.W.: Abstract symmetric boundary conditions. Trans. Am. Math. Soc. 45(3), 369-442 (1939)

7. de Branges, L.: Some Hilbert spaces of entire functions IV. Trans. Am. Math. Soc. 105, 43-83 (1962)

8. de Snoo, H., Winkler, H.: Canonical systems of differential equations with self-adjoint interface conditions on graphs. Proc. R. Soc. Edinb. Sect. A 135(2), 297-315 (2005)

9. Derkach, V.: Boundary triplets, Weyl functions, and the KreĬn formula. In: Alpay, D. (ed.) Operator Theory, pp. 183-218. Springer, Basel (2015)

10. Derkach, V.A., Malamud, M.M.: Generalized resolvents and the boundary value problems for Hermitian operators with gaps. J. Funct. Anal. 95(1), 1-95 (1991)

11. Derkach, V.A., Malamud, M.M.: The extension theory of Hermitian operators and the moment problem. J. Math. Sci. 73(2), 141-242 (1995)

12. Dym, H., McKean, H.P.: Gaussian processes, function theory, and the inverse spectral problem. Probability and Mathematical Statistics, vol. 31. Academic Press, New York-London (1976)

13. Eckhardt, J.: An inverse spectral problem for a star graph of Krein strings. J. Reine Angew. Math. 715, 189-206 (2016)

14. Fattorini, H.O.: Second Order Linear Differential Equations in Banach Spaces, North-Holland Mathematics Studies, vol. 108. North-Holland Publishing Co., Amsterdam (1985)

15. Fleige, A., Winkler, H.: An indefinite inverse spectral problem of Stieltjes type. Integral Equ. Oper. Theory 87(4), 491-514 (2017)

16. Güneysu, B., Keller, M., Schmidt, M.: A Feynman-Kac-Itô formula for magnetic Schrödinger operators on graphs. Probab. Theory Relat. Fields 165(1-2), 365-399 (2016)

17. Hassi, S., De Snoo, H., Winkler, H.: Boundary-value problems for two-dimensional canonical systems. Integral Equ. Oper. Theory 36(4), 445-479 (2000)

18. Kac, I.S., Kreĭn, M.G.: On the spectral functions of the string. Am. Math. Soc. Transl. (2) 103, 19-102 (1974)

19. Kac, I.S., Kreı̆n, M.G.: R-functions - Analytic functions mapping the upper half-plane into itself. Am. Math. Soc. Transl. (2) 103, 1-18 (1974)

20. Kac, I.S., Pivovarchik, V.N.: On the density of the mass distribution of a string at the origin. Integral Equ. Oper. Theory 81(4), 581-599 (2015)

21. Kato, T.: Perturbation Theory for Linear Operators, Classics in Mathematics. Springer, Berlin (1995). Reprint of the 1980 edition

22. Kočuber̆, A.N.: Extensions of symmetric operators and of symmetric binary relations. Math. Zametki 17, 41-48, (1975). Engl. transl. Math. Notes 17(1), 2528 (1975)

23. Kramar Fijavž, M., Mugnolo, D., Sikolya, E.: Variational and semigroup methods for waves and diffusion in networks. Appl. Math. Optim. 55(2), 219-240 (2007)

24. Kreı̆n, S.G.: Linear Differential Equations in Banach Space, Translations of Mathematical Monographs, vol. 29. American Mathematical Society, Providence (1971)

25. Kurasov, P.: Schrödinger operators on graphs and geometry. I. Essentially bounded potentials. J. Funct. Anal. 254(4), 934-953 (2008)

26. Kurasov, P.: Quantum Graphs: Spectral Theory and Inverse Problems. Oper. Theory Adv. Appl. (2018) (to appear, Birkhäuser Basel)

27. Langer, H., Schenk, W.: Generalized second-order differential operators, corresponding gap diffusions and superharmonic transformations. Math. Nachr. 148, 7-45 (1990)

28. Langer, H., Textorius, B.: On generalized resolvents and $Q$-functions of symmetric linear relations (subspaces) in Hilbert space. Pac. J. Math. 72(1), 135-165 (1977)

29. Lenz, D., Pankrashkin, K.: New relations between discrete and continuous transition operators on (metric) graphs. Integral Equ. Oper. Theory 84(2), 151-181 (2016)

30. Pivovarchik, V., Rozhenko, N., Tretter, C.: Dirichlet-Neumann inverse spectral problem for a star graph of Stieltjes strings. Linear Algebra Appl. 439(8), 2263-2292 (2013)

31. Pivovarchik, V., Tretter, C.: Location and multiplicities of eigenvalues for a star graph of Stieltjes strings. J. Differ. Equ. Appl. 21(5), 383-402 (2015)

32. Pivovarchik, V., Woracek, H.: Sums of Nevanlinna functions and differential equations on star-shaped graphs. Oper. Matrices 3(4), 451-501 (2009) 
33. Post, O.: Spectral Analysis on Graph-Like Spaces. Lecture Notes in Mathematics vol. 2039. Springer, Berlin (2012)

34. Schmüdgen, K.: Unbounded Self-Adjoint Operators on Hilbert Space, Graduate Texts in Mathematics, vol. 265. Springer, Dordrecht (2012)

35. Simonov, S., Woracek, H.: Spectral multiplicity of selfadjoint Schrödinger operators on star-graphs with standard interface conditions. Integral Equ. Oper. Theory 78(4), 523-575 (2014)

36. Vasil'ev, V.V., Piskarev, S.I.: Differential equations in Banach spaces. II. Theory of cosine operator functions. J. Math. Sci. (N.Y.) 122(2), 3055-3174 (2004)

37. von Below, J.: Sturm-Liouville eigenvalue problems on networks. Math. Methods Appl. Sci. 10(4), 383-395 (1988)

38. Winkler, H.: The inverse spectral problem for canonical systems. Integral Equ. Oper. Theory 22(3), 360-374 (1995) 